\title{
Efficient Wireless Video Communication using Sophisticated Channel Coding and Transmitter Diversity Gain Technique
}

Nasu Minallah ( $\sim$ minallah.nasru@gmail.com )

University of Engineering and Technology

Khadem Ullah

University of Engineering and Technology

Imran Ullah Khan

Harbin Engineering University https://orcid.org/0000-0002-3268-4844

Khurram Shahzad Khattak

University of Peshawar

\section{Research}

Keywords: sample, article, author Recursive Systematic Convolution (RSC) codes, Sphere Packing (SP) Modulation, BER reduction, Differential Space Time Spreading (DSTS), Extrinsic Information Transfer (EXIT) Chart

Posted Date: July 6th, 2020

DOl: https://doi.org/10.21203/rs.3.rs-35714/v1

License: (c) (1) This work is licensed under a Creative Commons Attribution 4.0 International License. Read Full License 


\title{
RESEARCH
}

\section{Efficient Wireless Video Communication using Sophisticated Channel Coding and Transmitter Diversity Gain Technique}

\author{
Nasru Minallah ${ }^{1 *}$, Khadem Ullah ${ }^{2}$ \\ and Imran Ullah Khan ${ }^{3}$
}

${ }^{*}$ Correspondence:

n.minallah@uetpeshawar.edu.pk

${ }^{1}$ Department of Computer

Systems Engineering, University of Engineering and Technology

Peshawar, 25000, Peshawar,

Pakistan

Full list of author information is

available at the end of the article

${ }^{\dagger}$ Equal contributor

\begin{abstract}
This article investigate the performance of various sophisticated channel coding and transmission schemes for achieving reliable transmission of H.264/AVC compressed video. The performance of the proposed schemes, namely Non-Convergent Coding (NCC), Non-Convergent Coding assisted with Differential Space Time Spreading (DSTS) and Sphere Packing (SP) modulation (NCDSTS-SP) and Convergent Coding assisted with Differential Space Time Spreading (DSTS) and Sphere Packing (SP) modulation CDSTS-SP, is analyzed using Bit Error Ratio (BER) and Peak Signal to Noise Ratio (PSNR) performance of the transmitted video stream. Channel codes incorporate artificial residual redundancy in the coded information bits, which is advantageous in the decoder side to overcome error effects and to accomplish the lowest desired BER. To cope with the very high compression ratio efficiency of the H.264/AVC video codec, our proposed system induces artificial redundancy in the compressed video bit-stream with the aid of Over Complete Mapping (OCM) and Recursive Systematic Convolution (RSC) channel codes, in order to improve the error resilience of the transmitted stream. Furthermore, overall BER reduction and improvement in objective quality performance is achieved using sophisticated transceiver design consisting of the advanced Sphere Packing (SP) modulation technique assisted by Differential Space Time Spreading (DSTS). The performance of the Iterative Soft Bit Source Decoding (SBSD) and channel decoding is analyzed using various error protection setups by allocating persistently constant overall bit rate budget. Additionally, the Iterative behavior of the SBSD assisted Recursive Systematic Convolution (RSC) code is analyzed with the aid of Extrinsic Information Transfer (EXIT) Chart. Moreover, it is observed from the experimental results that the sophisticated system design of CDSTS-SP outperforms its counterpart in terms of BER and PSNR. More specifically NCDSTS-SP results in PSNR gain of $6 \mathrm{~dB}$ and CDSTS-SP results in PSNR gain of $28 \mathrm{~dB}$ for $E_{b} / N_{0}$ value of $10 \mathrm{~dB}$, with reference to bench marker system design of NCC.
\end{abstract}

Keywords: sample; article; author Recursive Systematic Convolution (RSC) codes; Sphere Packing (SP) Modulation; BER reduction; Differential Space Time Spreading (DSTS); Extrinsic Information Transfer (EXIT) Chart

\section{Introduction}

In wireless communication systems due to the increasing interest in multimedia applications, video transmission becomes one of the most interesting and pragmatic 
research field for the next generation. High Definition (HD) videos required higher bandwidth on communication channels even with the employment of video compression. For one pixel representation of a gray scale and color video, 8 and 24 bits are used correspondingly. A total of $944 \mathrm{Mbps}$ is required to display one frame of simple colored TV video with a resolution of $1024 \times 768$ pixels, frame rate of $25 \mathrm{fps}$ [1]. Typically, bandwidth requirement is reduced by encoding the video content through video coding standards such as H.264/AVC, to transport over the network. The compressed video still required bandwidth in kbps or Mbps. Major factors affecting the multimedia communication are autonomous nature of Internet, inherent instability, deficiency in service guarantee and bandwidth instability. Shannon proposed the basics of channel capacity bounds over noisy channel, which also identifies the error-resilient features of the communication systems [2]. Shannon limit predicts the highest data rate and specify the bound on error-free information to be achieved on specific bandwidth over a noisy communication channel upon the addition of redundant bits to the messages to be transmitted. The first forward error correction block code for single error correction was the Hamming code proposed in [3]. Elias was the first who discovered error-correcting convolutional codes in [4], in which the encoding dependencies shifted from finite-length segments to encoding dependencies that exist over the entire block. The authors in [5] used convolutional codes for burst of error correction. In [6,7] different algorithms were proposed for decoding the convolutional codes. The Viterbi Algorithm (VA) proposed in [8] is a significant achievement ever in the history of convolutional correction codes. VA primarily works on finding the closest sequence of the transmitted information bits and results in minimum possible BER. Authors in [9] proposed the Maximum A Posteriori (MAP) algorithm which gives better performance in terms of BER and defeats VA but its infrequent usage is due to offering higher complexity. Turbo codes are first presented in $[10,11]$, which comprises the concatenation of two Recursive Systematic Convoluational (RSC) codes together attached with an interleaver. Turbo codes are also used in third-generation (3G) mobile radio systems due to its substantial improvement [12]. In turbo codes, an iterative algorithm is used at the decoder to extract the transmitted information bits. The authors proposed Soft Output Viterbi Algorithm (SOVA) in [13]. They modified the VA in such a way that it gives the most probable transmitted sequence in a Markov finite state chain along with the reliability or posteriori information. Koch et.al in [14] proposed the Max Log MAP algorithm for turbo decoder with lower complexity than SOVA. The authors in [15] evaluates the performance of the Joint Source Channel codes and finds that it could be jointly optimized as one pair for achieving a lower BER. The proposed algorithm extended curve-fitting optimal design criteria and a procedure for finding the code pair with the corresponding threshold decoding. Communication over the Rayleigh Fading channel, where a signal is severely attenuated when transmitted over fading channel is another challenging task. Mostly, diversity gain techniques are used to overcome the problem in which several copies of the same signal are transmitted at each instance of time, assuming that one of these signals is not severely attenuated. In Multiple Input Multiple Output (MIMO) scheme multiple transmitter and receiver antennas are deployed to achieve better performance at the receiver, but its achievement become limited due to its requirement of Channel State Information (CSI) [16]. Every signal has their own impulse response which makes the 
achievable performance minimum and can be enhanced by designing sophisticated transceiver. The capacity of MIMO systems linearly increases with increasing the number of transmitter antennas, provided that the number of receiver antennas is greater than the transmitter antennas [17]. Space Time Coding (STC) [18] provides the diversity gain but the main issue in employing these codes is the estimation of the multipath channel effect. In [19], the authors proposed a low complexity transmitter diversity technique which provides similar performance for two transmitters $(\mathrm{Tx})$ with one receiver antenna $(\mathrm{Rx})$, to the performance of maximal-ratio combining (MRCC) technique with one Tx and two Rx. The differential coding scheme can be a good choice for attaining diversity gain in which there is no channel impulse response estimation required. In [20], Differential Phase Shifting Keying (DPSK) is proposed for a single transmitter antenna, which gives the independence from the channel estimation. The nature of a channel in DPSK modulation for a period of two symbols is assumed to be constant, therefore two symbols are transmitted each time. The receiver then detects by observing the two successive symbols. Differential Space Time Spreading (DSTS) [16] tends to overcome the channel estimation dependency for each individual MIMO link and hence results in differential detection with a lower complexity algorithm. There are two main stages in DSTS, first the symbols that are modulated by SP Modulation [21] are differentially encoded and then secondly is spread out by spreading codes. A delightful source codec candidate for wireless video communication is the highest compression standard H.264/AVC [22]. The dramatic improvement in its compression ratio is due to using the Variable Length Coding (VLC) and motion or displacement vectors in pixel prediction techniques. The high compressed bit-streams are more vulnerable to transmission errors as compared to uncompressed or compressed by a lower compression ratio standard. The single error in transmission may furnish the correct decoding of succeeding codewords harder. Additionally, the error is propagated to the neighboring data blocks in predictive algorithms, which makes the transmission of compressed video a challenging task. Error resilient schemes are used to weaken this error sensitivity problem. These schemes reduce the compression efficiency at the cost of increased computational complexity. Several other error resilient techniques for H.264/AVC exist [23] which include layered video coding using Unequal Error Protection (UEP).

Taking in mind the above background, we propose an arrangement for the H.264/AVC compressed video bitstream transmission through Soft-Bit Source Decoding (SBSD) scheme. Artificial redundancy is generated in the transmitter side using two different combination of OCM and RSC encoder while keeping the overall bitrate constant. The redundancy is iteratively utilized in the decoder side for the enhancement of high BER performance. As already discussed that its hard to estimate an impulse response for each individual MIMO link in Rayleigh fading channel due to experiencing different fading. In order to further improve the performance of the system, a transmitter diversity gain technique is incorporated, such that the coded video bitstream is passed to DSTS and SP modulation which overcome channel estimation dependency. The motivation of this research article was to investigate the mechanism of performance improvement using iterative coding, while employing convergence capable inner and outer code, transmitter diversity gain and its impact on the performance of highly compression efficient coded video stream. 
The reset of the proposed manuscript has been structured as follows. Section 2 provides a detailed analysis of the proposed system models while the video encoder/decoder H.264/AVC compression standard is presented in section 3. Random variables and notations that are used in our simulation are explained in section 4 . Section 5 provides the description of RSC codes. Overview of OCM and EXIT chart analysis is presented in Section 6 while section 7 presents the performance analysis of our propose simulation. Finally, a conclusion of the resultant work is provided in Section 8.

\section{Proposed System Model}

Typically, the primitive of the transmitter block comprises discrete input, encoder, digital modulator, and the output. Channel encoder is operating for the removal of error effects by adding some redundancy through a controlled methodology in the information bits. Channel flexibility takes place by manipulating RSC codes as a constituent channel codes to efficiently operate in AWGN and as well in Rayleigh fading channels. The proposed system model comprises three video phone arrangements as shown in figure 1 and the specifications of the transceivers are given in Table 1 and 5 . All the transceivers follow the same mechanism and are only different by using different modulation schemes and arrangements of inner and outer code rates. In the proposed scheme, video is initially compressed at the transmitter side by using the international compression standard H.264/AVC video codec with the parameters settings of the source encoder as given in Table 4 . The different building blocks of the proposed system models are as follows.

\subsection{Source channel encoding:}

The compressed video bit-stream comprises three partitions types $\mathrm{A}, \mathrm{B}$, and $\mathrm{C}$ of all the slices per frame. The bit-stream $x_{i}$ is then mapped(OCM) to bit-string $x_{i}^{\prime}$ using a special bitmapping scheme, referred to as Over-complete Mapping (OCM), where we have $i=1,2,3 \ldots n$. Thereafter the mapped bit string $x_{i}^{\prime}$ is interleaved to conquer the burst of error into $\bar{x}_{i}$ using the bit interleaver (П). The interleaved sequence $\bar{x}_{i}$ is then passed to the RSC encoder which encodes the sequence by different code rates as shown in table 1. Our proposed NCC an NCDSTS-SP schemes employ Rate-1 OCM, presented in Table-2, as inner code while Rate-1/3 RSC code is employed as outer channel code. Contrary to this and inorder to improve the convergence behaviour of our employed iterative coding, CDSTS-SP scheme employ Rate-3/4 OCM, presented in Table-2, as inner code while Rate-4/9 RSC code is employed as outer channel code. The channel coded output from this block is forwarded to the next block for further processing.

\subsection{Modulation and Transmission:}

The encoded bit-stream received from the source channel encoding block is then forwarded to modulation and transmission block. Using NCC scheme the received bit-stream is mapped to the stream of QPSK symbols. Afterwards, the Single Input Single Output (SISO) transmitter antenna is used to transmit the QPSK modulated Symbols over Rayleigh Fading channels.

While using NCDSTS-SP and CDSTS-SP schemes the received bit-stream is mapped to the SP symbols $S_{i}$, where $i=0,1,2,3 \ldots L-1$ by SP mapper, L 


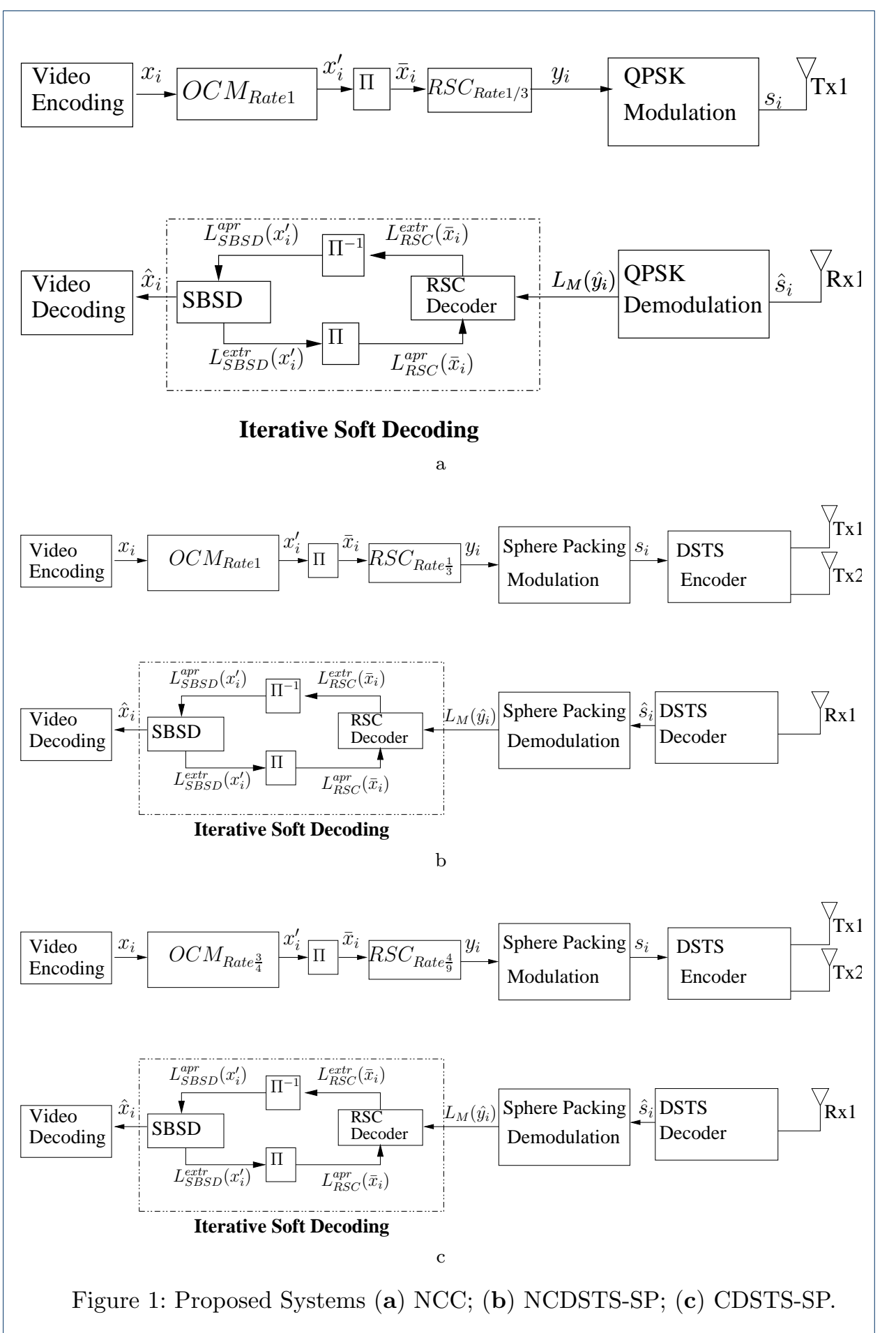

is the modulated symbols that represents the number of SP signaling elements [21]. Afterwards, the DSTS transmitter antenna is used to transmit the SP modulated Symbols over Rayleigh Fading channels. DSTS transceiver is liable to diversity gain in wireless network, reduced BER and accomplished a substantial video quality improvement. Due to embedding DSTS MIMO scheme, the proposed transceivers can 
Table 1: Parameters settings of the transceivers

\begin{tabular}{|l|l|l|l|}
\hline \multirow{2}{*}{ System } & \multicolumn{3}{c|}{ Parameters } \\
\cline { 2 - 4 } & NCC & NCDSTS-SP & CDSTS-SP \\
\hline Ourter Code & Rate-1 OCM & Rate-1 OCM & Rate-3/4 OCM \\
\hline Inner Code & Rate-1/3 RSC & Rate-1/3 RSC & Rate-4/9 RSC \\
\hline Modulation & QPSK & SP & SP \\
\hline MIMO Scheme & Nill & DSTS & DSTS \\
\hline Tx & 1 & 2 & 2 \\
\hline Rx & 1 & 1 & 1 \\
\hline Channel & Rayleigh & Rayleigh & Rayleigh \\
\hline Doppler Frequency & 0.01 & 0.01 & 0.01 \\
\hline
\end{tabular}

Table 2: Probabilities Of Symbols with OCM Rate-1 and Rate- $\frac{3}{4}$

\begin{tabular}{|c|c|c|}
\hline Symbols & OCM Rate- 1 & OCM Rate- $\frac{3}{4}$ \\
\hline 000 & 000 & 0000 \\
001 & 001 & 1001 \\
010 & 010 & 1010 \\
011 & 011 & 0011 \\
100 & 100 & 1100 \\
101 & 101 & 0101 \\
110 & 110 & 0110 \\
111 & 111 & 1111 \\
\hline
\end{tabular}

Table 3: Analysis of different sampling formats and video sequences

\begin{tabular}{|c|c|c|c|c|c|}
\hline Sampling format & $\begin{array}{l}\text { Video Sequence } \\
\text { Resolution }\end{array}$ & $\begin{array}{l}\text { Luminance }(\mathrm{Y}) \\
\text { Resolution }\end{array}$ & $\begin{array}{l}\text { Luminance } \\
\text { bits per frame }\end{array}$ & $\begin{array}{l}\text { Chrominance } \\
\text { (Cb \& Cr) } \\
\text { Resolution }\end{array}$ & $\begin{array}{l}\text { Chrominance } \\
\text { bits per frame }\end{array}$ \\
\hline \multirow{3}{*}{ YUV(4:4:4) } & QCIF & $176 \times 144$ & 608256 & $176 \times 144$ & 608256 \\
\hline & $\mathrm{CIF}$ & $352 \times 288$ & 2433024 & $352 \times 288$ & 2433024 \\
\hline & $4 \mathrm{CIF}$ & $704 \times 576$ & 9732096 & $704 \times 576$ & 9732096 \\
\hline \multirow{3}{*}{ YUV(4:2:2) } & QCIF & $176 \times 144$ & 405504 & $88 \times 144$ & 202752 \\
\hline & $\mathrm{CIF}$ & $352 \times 288$ & 1422016 & $176 \times 288$ & 811008 \\
\hline & $4 \mathrm{CIF}$ & $704 \times 576$ & 6488064 & $352 \times 576$ & 3244032 \\
\hline \multirow{3}{*}{ YUV(4:2:0) } & QCIF & $\overline{176 \times 144}$ & 304128 & $88 \times 72$ & 76032 \\
\hline & $\mathrm{CIF}$ & $352 \times 288$ & 1216512 & $176 \times 144$ & 304128 \\
\hline & $4 \mathrm{CIF}$ & $704 \times 576$ & 4866044 & $352 \times 288$ & 1216512 \\
\hline Video Sequence (VS) & Frames & Frame rate & \multicolumn{3}{|c|}{ Reason for a selection } \\
\hline AIYO & \multirow{3}{*}{45} & \multirow{3}{*}{$15 \mathrm{fps}$} & \multicolumn{3}{|c|}{ Low motion and dynamism } \\
\hline FOREMAN & & & \multirow{2}{*}{\multicolumn{3}{|c|}{$\begin{array}{l}\text { Medium motion and dynamism } \\
\text { High motion and dynamism }\end{array}$}} \\
\hline MOBILE & & & & & \\
\hline
\end{tabular}


be deployed in diverse environment for providing the same coverage and quality performance.

At the decoder side the received signal is first presented to the DSTS decoder and SP demapper which gives the Log-Likelihood Ratios (LLRs) value for the bitstream. The generated LLR values are forwarded to the next block for further processing.

\subsection{Iterative Soft Decoding:}

Subsequently, the extrinsic LLRs information received from the modulation and transmission block is then exchanged between the RSC decoders and SBSD in iterative fashion. The extrinsic information for Markov zero order model using SBSD can be found in [24]. The residual redundancy remains in M-ary symbols after the source encoding by H.264/AVC in term of non-uniform probability distribution $P\left[S_{n}(j)\right]$, where $S_{n}(j)=\left[S_{n}(1), S_{n}(2), S_{n}(3) \ldots S_{n}(M)\right]$ refer to table 2 . The results in table 2 is accomplished by assigning 3 bits/symbols to the H.264/AVC encoded bit-stream, i.e. $\left[b_{1}(1), b_{1}(2), b_{1}(3) ; b_{2}(1), b_{2}(2), b_{2}(3) \ldots b_{j}(3)\right]$. The channel output symbol is generated by the following expression, provided that the bits are independent of each other. Eventually, the channel output information is combined with the A-priori knowledge of that corresponding symbol to achieve the resultant LLR extrinsic value, as expressed below.

$$
P\left[\hat{y}_{\tau} \mid y_{\tau}\right]=\prod_{k=1}^{K} P\left[\hat{y}_{\tau}(k) \mid y_{\tau}(k)\right],
$$

Where $\hat{y}_{\tau}$ is the received $K$-bit sequence on transmission of $y_{\tau}$. For a specific bit $\left[y_{\tau}(\lambda)\right]$, the output extrinsic channel information $P\left[\hat{y}_{\tau}^{[\text {ext }]} \mid y_{\tau}^{[\text {ext }]}\right]$ can be achieved as follow.

$$
P\left[\hat{y}_{\tau}^{[e x t]} \mid y_{\tau}^{[e x t]}\right]=\prod_{k=1, k \neq \lambda}^{K} P\left[\hat{y}_{\tau}(k) \mid y_{\tau}(k)\right] .
$$

Eventually, the channel output information is combined with the A-priori knowl-

edge of that corresponding symbol to achieve the resultant LLR extrinsic value, as expressed below.

$$
\begin{aligned}
& \operatorname{LLR}\left[y_{\tau}(\lambda)\right]= \\
& \log \left(\frac{\sum_{y_{\tau}^{[e x t]}} P\left[y_{\tau}^{[\text {ext }]} \mid y_{\tau}(\lambda)=+1\right] \cdot P\left[\hat{y}_{\tau}^{[\text {ext }]} \mid y_{\tau}^{[\text {ext }]}\right]}{\sum_{\left.y_{\tau}^{[\text {ext }}\right]} P\left[y_{\tau}^{[\text {ext }]} \mid y_{\tau}(\lambda)=-1\right] \cdot P\left[\hat{y}_{\tau}^{[\text {ext }]} \mid y_{\tau}^{[\text {ext }]}\right]}\right) .
\end{aligned}
$$

\section{H.264 Video Coding Standard}

The international video compression standard, Advance Video Coding (AVC)/H.264 is one of the latest in a video compression standard sequence of [25-29]. This enhancement in video compression technology in the form of H.264/AVC, resulted 
in extension of video service delivery to various networks and applications. The coding architecture of H.264 consists of two basics layers i.e. Video Coding Layer (VCL) and Network Adaptation Layer (NAL). VCL provided efficient representation of video contents while NAL provides network friendly representation. H.264 generates video frame slices which are comprised of Macroblacks (MBs). Each slice consists a sequence of MBs with in the same slice group [30]. Macroblock coding are categorized into two modes i.e. inter and intra-coded. In inter-coding, each block is transmitted along with the displacement vector, which is already calculated from the motion compensation of the previously transmitted information. The displacement vector is the pointer to the previous image signal position stored in memory, as a reference at the receiver side. For decoding purposes, the prediction error is calculated and combined with the currently received information, from which the exact image is estimated. In intra-coding mode, the image is encoded without reference to the previously transmitted information bits. In addition to the significant compression efficiency, the H.264/AVC is designed in such a way which also offer error-resilient features. To reduce the effect of channel errors, H.264 uses error resilient data partitioning (DP). DP generate three different types of partitions per slice which are type A, B and C stream. The importance of these partitions is variable in term of their information contents.

Table 4: Parameters settings of the source encoder

\begin{tabular}{|l|r|}
\hline Video Coding & Parameters \\
\hline \hline Compression standard & H.264/AVC \\
Date-Rate in Kbps & 64 \\
Frame-Rate in fps & 15 \\
Number of Slices Per frame & 9 \\
Macro-Blocks Per Slice & 11 \\
Macro-Blocks per frame in Intra-frame & 3 \\
Profile & Extended \\
IntraPeriod of I-pictures & 15 \\
PartitionMode & 3 Partitions per Slice \\
Entropy coding method & UVLC \\
SliceMode & Fixed \# of MBs in slice \\
\hline
\end{tabular}

- Partition A is the most important partition, comprising header information of each slice, motion vector, macroblock types and quantisation parameters. In-case, If partition A gets noisy, then the complete packet including partition $\mathrm{B}$ and $\mathrm{C}$ is dropped and as a result the entire block is marked as distorted. Therefore, the decoder will then attempt an error concealment technique by using the formerly transmitted video information.

- Partition B comprises from intra Coded Block Patterns (CBP) bits and also MB coefficients. The impact of error propagation can be reduced by encoding specific image areas in intra-coded by switching from inter-frame prediction. A very limited part of the image is encoded in intra-frame mode. Therefore type $\mathrm{B}$ in the entire encoded slice contains the lowest number of bits.

- Partition $\mathrm{C}$ contains inter frame motion compensated residual (MCR) bits and inter frame CBP. Additionally, this partition also contains the MCR bits for macroblocks encoded by intra frame prediction of H.264.

Therefore, the importance of partition $\mathrm{A}$ is understandable. Both the partitions B and $\mathrm{C}$ are dependent on $\mathrm{A}$ and cannot be decoded in absence of partition $\mathrm{A}$. The intra frame macroblock update is added to the reconstructed frame, in case if A and 
B both partitions are uncorrupted, while in the presence of partition $\mathrm{C}$ the MCR is augmented to the motion compensated block.

Table 5: Code rate and modulation technique in the proposed models.

\begin{tabular}{|l|l|l|l|}
\hline \multirow{2}{*}{ Scheme } & \multicolumn{2}{|c|}{ Bit rate } & \multirow{2}{*}{ Modulation } \\
\cline { 2 - 3 } & Outer Code & Inner Code & \\
\hline NCC & Rate-1 OCM & Rate-1/3 RSC code & QPSK \\
\hline NCDSTS-SP & Rate-1 OCM & Rate-1/3 RSC code & Sphere Packing \\
\hline CDSTS-SP & Rate-3/4 OCM & Rate-4/9 RSC code & Sphere Packing \\
\hline
\end{tabular}

\section{Random Variables}

In our simulation, the following random variables and notations are used in the proposed systems as defined in [31].

Basics of communication model consist of sender, receiver, message, communication channel and protocol. The underlined concept of the communication channel is defined as, the emitted electromagnetic waves do not reach directly to the receiving antenna due to obstacles producing the multi-path channel effect. The received signal is not in the form of the actual transmitted signal but it is the incorporation of its reflected, scattered and diffracted parts from walls, trees and buildings. The received signal consist of an infinite sum of the replica of the transmitted signal which is delayed, attenuated, phase-shifted and influencing each other. The superposition can be either constructive or destructive depending on the phase of each comprise signal. When the transmitter or the receiver is moving then the Doppler spread or frequency shifting occur. This type of channel is characterized by the Rayleigh fading model.

Rayleigh random variable $\mathrm{X}$ is defined as, the square root of independent and identically distributed Gaussian random variables $X_{1}$ and $X_{2}$ each having zero mean and $\sigma^{2}$ variance with two degree of freedom [31].

$$
X=\sqrt{X_{1}^{2}+X_{2}^{2}}
$$

The probability distribution function (PDF) of a random Rayleigh fading variable having mean equal to $2 \sigma^{2}$ is given by the following equation.

$$
P(X)=\left\{\begin{array}{cc}
\frac{X}{\sigma^{2}} e^{\frac{-X^{2}}{2 \sigma^{2}}} & X>0 \\
0 & \text { otherwise }
\end{array}\right.
$$

The mean and variance of the Rayleigh fading variable is:

$$
\begin{aligned}
& E[X]=\sigma \sqrt{\frac{\pi}{2}} \\
& V A R[X]=\left(2-\frac{\pi}{2}\right) \sigma^{2}
\end{aligned}
$$

Integrating the $\mathrm{PDF}$ gives the $\mathrm{CDF}$ of a Rayleigh variable i.e.

$$
F[X]=\left\{\begin{array}{cc}
1-e^{\frac{-X^{2}}{2 \sigma^{2}}} & X>0 \\
0 & \text { otherwise }
\end{array}\right.
$$


Lognormal distribution variable $(\mathrm{Y})$ is used for modeling the shadowing effect of the signal due to large blocks and building in mobile communication. This variable can be related to a new variable $\mathrm{X}$ through the following equation.

$$
Y=\ln X \vee X=e^{Y}
$$

Then the PDF of $\mathrm{X}$ is:

$$
P(X)=\left\{\begin{array}{cc}
\frac{1}{\sqrt{2 \pi \sigma^{2}} X} e^{\frac{-(\ln X-m)^{2}}{2 \sigma^{2}}} & X \geq 0 \\
0 & \text { otherwise }
\end{array}\right.
$$

Where $\mathrm{m}$ and $\sigma$ represents the mean and variance respectively.

$$
\begin{aligned}
& E[X]=e^{\frac{m+\sigma^{2}}{2}} \\
& V A R[X]=e^{2 m+\sigma^{2}}\left(\sigma^{2}-1\right)
\end{aligned}
$$

The Gaussian random variable is described in terms of two parameter $m \in \mathbb{R}$ and $\sigma>0$ by the PDF given as:

$$
P[X]=\frac{1}{\sqrt{2 \pi \sigma^{2}}} e^{\frac{-(X-m)^{2}}{2 \sigma^{2}}}
$$

The shorthand form of a Gaussian variable is $N\left(m, \sigma^{2}\right)$ to denote the PDF of a random variable. The mean and variance is given as:

$$
\begin{aligned}
& E[X]=m \\
& V A R[X]=\sigma^{2}
\end{aligned}
$$

Standard normal is achieved when the Gaussian variable has mean $=0$ and $\sigma=1$. Q function is closely related to this variable which can be defined as:

$$
P[N(0,1)>X]=\frac{1}{\sqrt{2 \pi}} \int_{0}^{\infty} e^{\frac{-t^{2}}{2}} d t
$$

The cumulative distribution functions of a Gaussian random variable is given by the following expression:

$$
\begin{aligned}
& F[X]=\int_{-\infty}^{X} \frac{1}{\sqrt{2 \pi \sigma^{2}}} e^{\frac{-(X-m)^{2}}{2 \sigma^{2}}} d t \\
& F[X]=1-\int_{X}^{\infty} \frac{1}{\sqrt{2 \pi \sigma^{2}}} e^{\frac{-(X-m)^{2}}{2 \sigma^{2}}} d t
\end{aligned}
$$




\section{Recursive Systematic Convolutional Codes Performance Parameters:}

Recursive Systematic Convolution (RSC) Codes is a class of error-correcting codes which is very important in coding theory due to its tremendous performance for sequential codes and ease of implementation. The characteristic of Convolution codes having constraint length $\mathrm{v}$, can be specified by its generator polynomial function (where $\mathrm{i}=1,2, \ldots \mathrm{n}$ generator polynomials) by the following equations [32].

$$
G^{(i)}(D)=g_{0}^{(i)}+g_{1}^{(i)} D+g_{2}^{(i)} D^{2} \ldots g_{(v-1)}^{(i)} D^{(v-1)}
$$

The D-transform input polynomial expression of Convolution code is given as:

$$
U_{n}^{\prime}(D)=u_{0}^{\prime}+u_{1}^{\prime} D+\ldots u_{n}^{\prime} D^{n}
$$

The encoder outputs for each individual bit-stream sequence can be obtained from the following equation.

$$
\begin{aligned}
& Y^{(i)}(D)=G^{(i)}(D) \cdot U_{n}^{\prime}(D) \\
& =Y_{0}^{(i)}+Y_{1}^{(i)} D+Y_{2}^{(i)} D^{2}+\ldots \ldots+Y_{v+n-1}^{(i)} D^{v+n-1}
\end{aligned}
$$

Where

$$
\begin{aligned}
& Y_{t}^{(i)}=\sum_{m=0}^{v-1} u(t-m)^{\prime} \cdot G_{m}^{(i)} \\
& Y_{t}^{i}=u_{t}^{\prime} g_{0}^{(i)} \text { XOR } u_{(t-1)}^{\prime} g_{1}^{(i)} \text { XOR }
\end{aligned}
$$

$$
u_{(t-2)}^{\prime} g_{2}^{(i)}+\ldots X O R u_{(t-v+1)}^{\prime} g_{(v-1)}^{(i)}
$$

In convolutional codes, there are $\mathrm{k}$ information bits infiltrate to the encoder and generates $n$ binary outputs at each instance of time. The $n$ outputs of the encoder generally corresponds to the generator polynomials. The next state of encoder and the output is depends on the current input as well as on instance of time. As a design example, consider the generator polynomials $G_{1}=(1,1,1)$ and $G_{2}=(1,1,0)$. The corresponding state diagram with the specified initial state is represented in Figure 2. For the state machine diagram description, a visualization for a state, its input and output values is specified in Figure 2. For the initial state 00, if the input 0 is fed in, the state remains as it. It is depicted by the notation 0/00 (input/output) in the diagram. In a similar fashion if we input 1, the state changes to 10 giving the output of 11 , represented as $1 / 11$ and the arrow directing towards the state 10 . The trend for all the other states is carried with the same convention and completed accordingly. The code rate $R_{c}$ of a convolutional codes is the ratio b/w the input $\mathrm{k}$ information bits infiltrate at a single time to the encoder and the binary outputs symbols $n$ to that input.i.e.

$$
R_{c}=\frac{k}{n}
$$




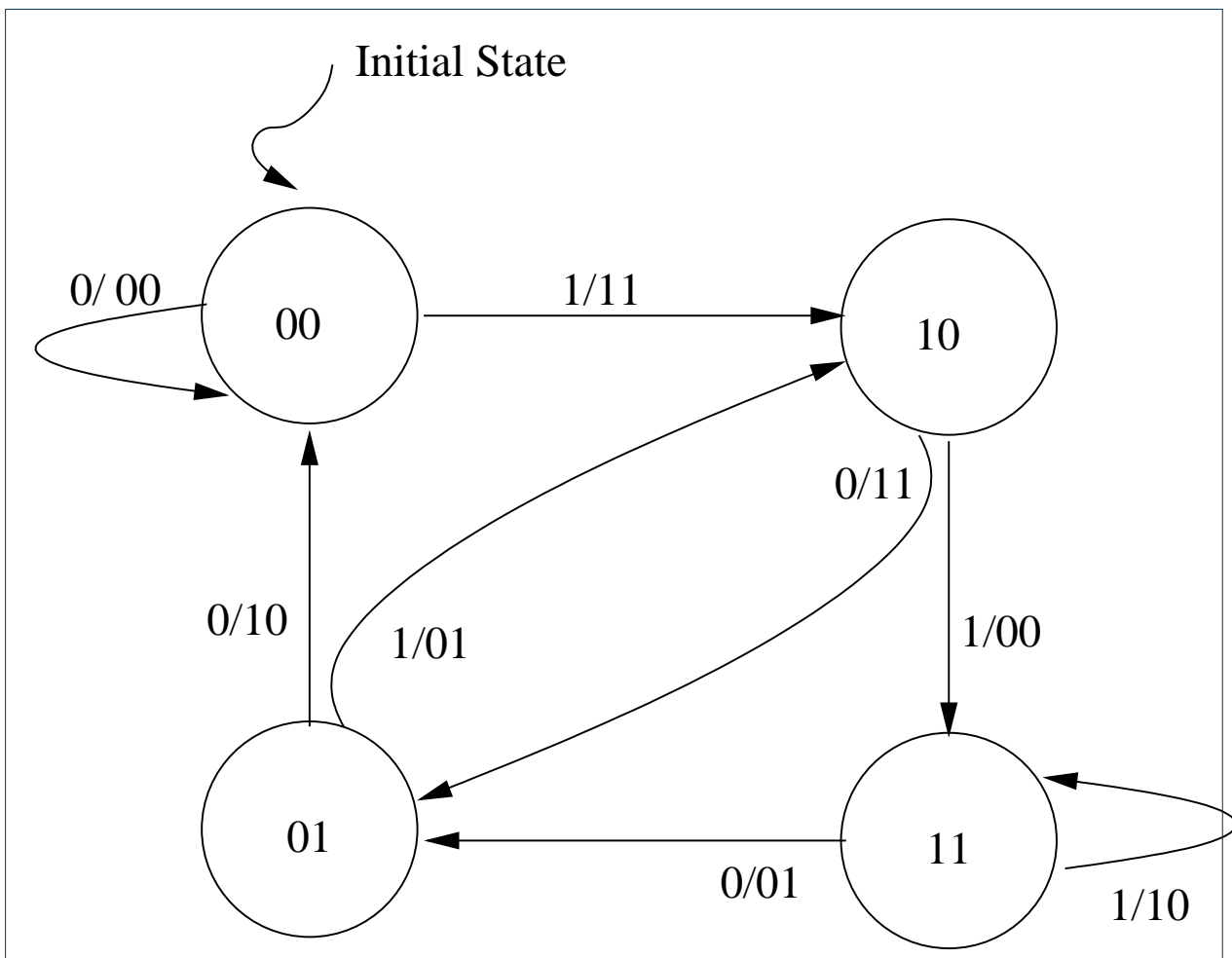

Figure 2: State Machine Diagram of Convolutional Coding

The unit of code rate is bits per transmission and represents the number of symbols sent in a single transmission. Typically the number of output bits is greater than the number of input bit which always leave the code rate less than 1 . The numbers of M-ary symbols transmitted per codeword L given by the following equation.

$$
L=\frac{n}{\log _{2} M}
$$

Where $\mathrm{n}$ is the length of codeword and $\mathrm{M}$ is the constellation size. The transmission time for $\mathrm{k}$ information bits is $T=L T_{s}$ when the symbol duration is $T_{s}$. We can derive an expression for the transmission $\operatorname{rate}(\mathrm{R})$ such as:

$$
R=\frac{k}{L T_{s}}
$$

Putting the value of $\mathrm{L}$ in the above equation.

$$
\begin{aligned}
& R=\frac{k * \log _{2} M}{n * T_{s}} \\
& R=R_{c} \frac{\log _{2} M}{T_{s}} b p s
\end{aligned}
$$

The bandwidth efficiency or spectral bit rate $(r)$ is the ratio of the bit rate of its encoding scheme to that of its bandwidth and can be used to calculate the 
bandwidth efficiency.

$$
r=\frac{R(b p s)}{W(H z)}
$$

The value of the spectral bit rate shows how much the system is efficient. The system will be transmitting at each hertz with higher bit rate and since will be more bandwidth efficient scheme if its have a larger value of spectral bit rate. The signal with bandwidth $\mathrm{W}$ can be reconstructed at the receiver if we sample the signal with a rate not less than the sampling rate of $2 \mathrm{~W}$ per second. The degree of freedom or dimensionality with duration $\mathrm{T}$ and bandwidth $\mathrm{W}$ can be expressed by the following expression.

$$
N=2 W T
$$

The minimum bandwidth requirement for the transmission can be expressed as:

$$
W=\frac{N}{T_{s}}
$$

Putting the value of $T_{s}$

$$
W=\frac{R N}{2 R_{C} \log _{2} M} \text { bits/sec }
$$

The pioneer source and channel code equations as defined in [31] were utilized for a new system model and our work focused on the improvements in performance of source and channel codes using the appropriates code rates for achieving perfect convergence of Iterative source channel-decoding and additionally by introducing SP Modulation.

\section{Iterative source decoding using OCM}

Natural redundancy residue in the encoded bit stream $x_{i}$ is used in conventional SBSD as source of performance to obtain the extrinsic information. The achievable performance in video bit stream is limited, when coding standard H.264/AVC used as encoder, which remove most of the residual redundancy. Therefore, the proposed scheme uses over complete mapping to generate artificial redundancy in coded video bit stream. To extract extrinsic information, the ingredient inner and outer decoder of iterative channel decoding provides sufficient information to each other in each iteration using the concept of interlacing.

EXIT chart operates on the special property of iterative decoder, which is convergence behavior as proposed by Stephen ten Brink [33], by exploiting the exchange of input and output mutual information. It is useful for serial, parallel and hybrid concatenated systems as well. Our proposed OCM is designed for employing this specific property of EXIT charts. If there is an open tunnel between the EXIT curves of the RSC and SBSD decoder, then the iterative decoding achieve extremely small decoded BER. Consequently, the above condition becomes satisfied when the 
EXIT curve intersects at a point $\left(I_{A}, I_{B}\right)=(1,1)$ and it require a hamming distance between any two codewords $\left(d_{H}, \min \right)=2$, which is briefly explained in [33]. This condition stimulates our OCM scheme that it is possible to find a code table that satisfies the above condition by complete search of all codewords having mapping rate reciprocal to that of code rate and having $\left(d_{H}, \min \right)=2$, as presented in Table-5. Based on this table, the EXIT curve gets closer to the perfect point of convergence i.e. reaching to $\left(I_{A}, I_{E}\right)=(1,1)$ of both the OCM and codec block, regardless of the shape of the Exit curve of the standalone encoder. As refer to table 2, the Exit optimized mapping ensures that the mapped m-bit symbols exhibit hamming distance of $\left(d_{H}, \min \right)=2$.

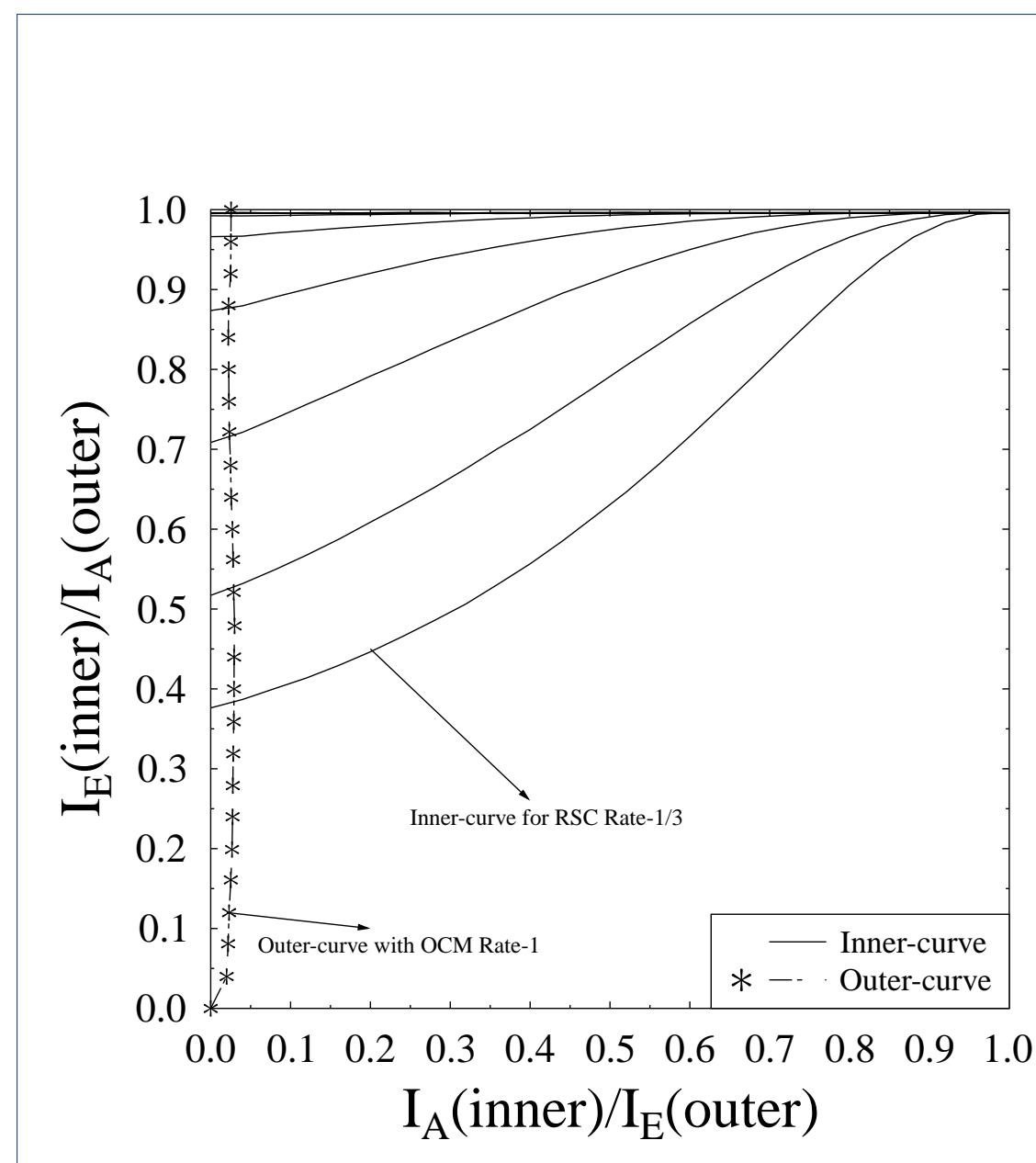

Figure 3: EXIT chart - Rate-1 OCM Outer Code and Rate-1/3 RSC Inner Code

Moreover, the EXIT curve of SBSD using OCM rate-1 inner code and RSC rate$1 / 3$ outer code is shown in Figure 3 . It can be observed that OCM rate- 1 is not capable to reach to the point of perfect convergence -i.e. to the rightmost corner at $\left(I_{A} ; I_{E}\right)=(1,1)$ and hence is not capable to exploit a beneficial advantage of iterative decoding. In contrast the EXIT curve of SBSD using OCM rate-3/4 inner code reaches the point of perfect convergence, which in combination with Rate- $4 / 9$ RSC code can get advantage of iterative decoding by iteratively exchanging extrinsic 


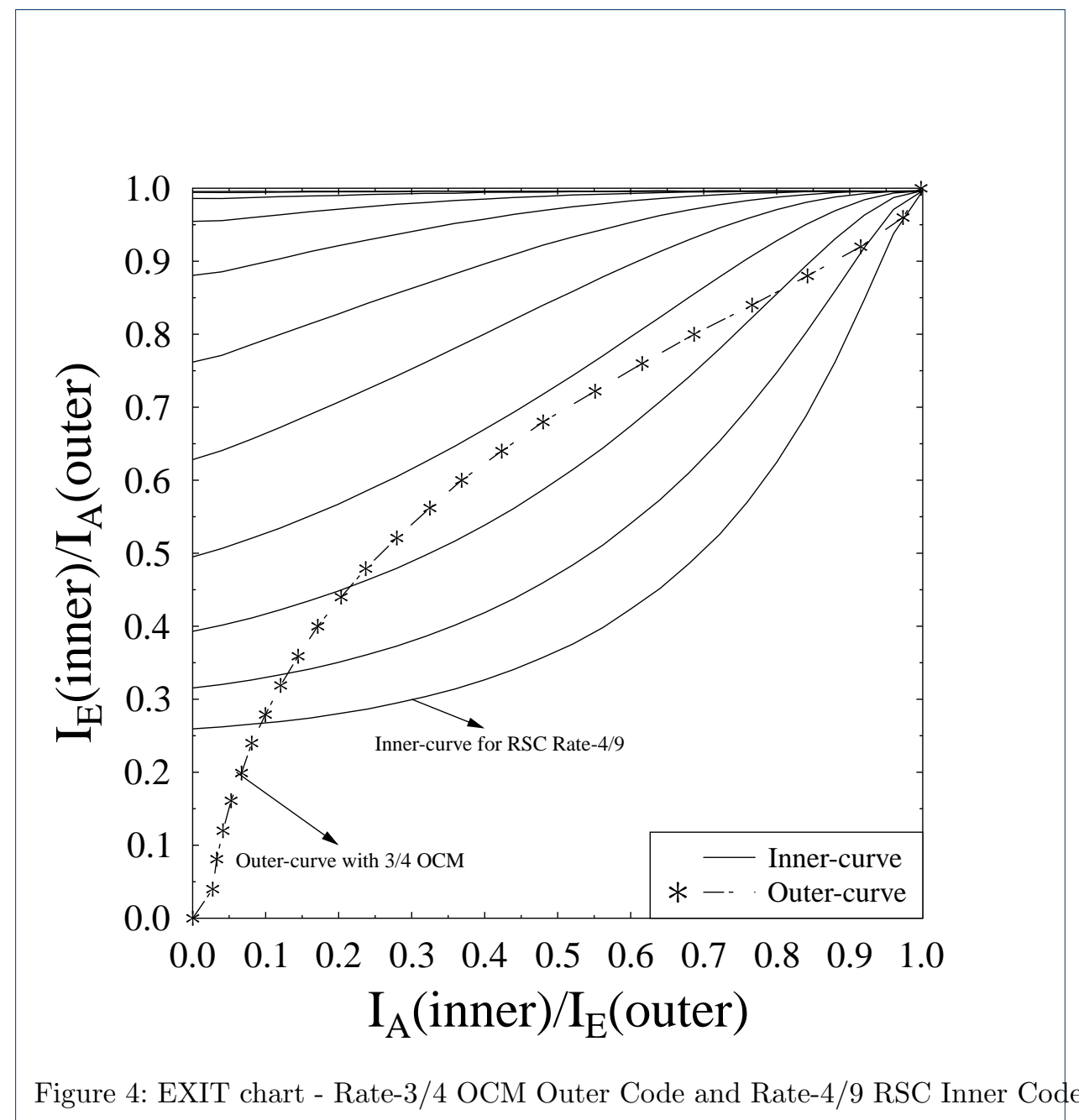

information to achieve very low BER refer to Figure 4. The EXIT Trajectory using OCM Rate-3/4 along with Rate-4/9 RSC code of CDSTS-SP at $E_{b} / N_{0}$ of $-0.3 d B$ is shown in Figure-5. It can be observed that due to convergence of inner and outer code to the point of perfect convergence - i.e. at $\left(I_{A} ; I_{E}\right)=(1,1)$, iterative decoding is capable to iteratively reach at the point of perfect convergence through an open-tunnel available at $-0.3 d B E_{b} / N_{0}$ and above.

\section{Simulations results and analysis}

This section provides a detailed performance analysis of the proposed system. The JM version 15 video codec developed by the joint video team (JVT) is used as a reference H.264 encoder. The proposed systems were simulated using IT++ signal processing and communications library, coded in $\mathrm{C}++$. To perform a fair analysis between the three schemes, several aspects of the proposed systems have been considered. Various sample of the diverse video sequences were considered and were provided as an input to the considered system models. Convergence behavior in iterative source channel decoding is specifically analyzed in the proposed work with different code rates of the inner and outer channel codes. In our simulation three 


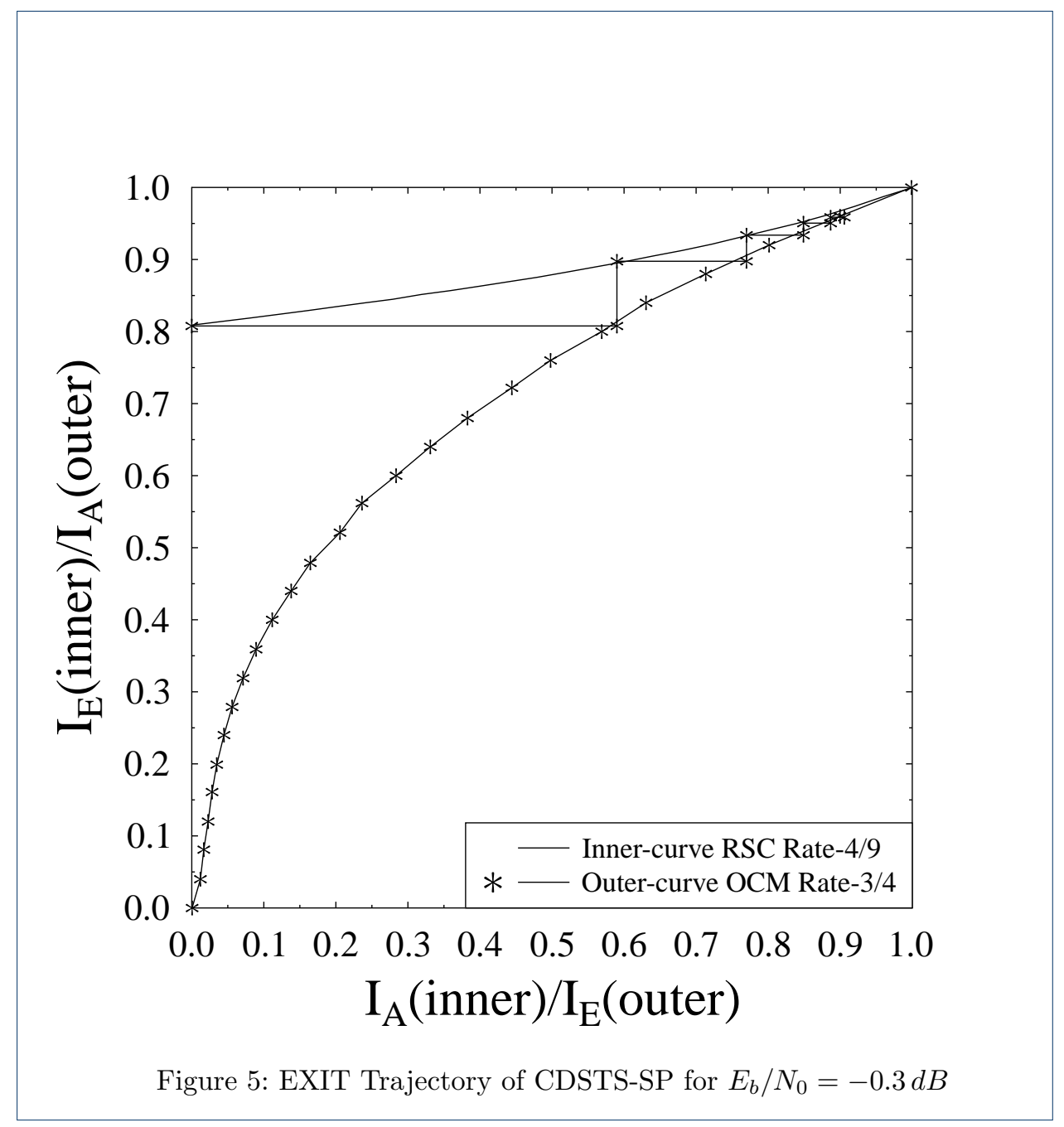

different video sequences -i.e. AKIYO, FOREMAN and MOBILE as shown in figure 6, each with three different resolution (CIF, QCIF and 4CIF) in YUV(4:2:0) format were used as testing sequences. Brief information about the video sequences used in the simulation experiments is as follows:

- Akiyo: This is a video of newscaster with extremely negligible movement and less details in the background.

- Foreman: This is a video of a foreman with rapidly moving face, extensive zoom out, average details and regular structures.

- Mobile: This is a video of a moving toy train with high details and dynamism.

The idea of using these different diverse video sequences with different video resolutions, level of motion and dynamism is to investigate the impact of video contents on its objective video quality while considering similar channel. As the extent of compression and robustness of the coded bit-stream is directly linked with its resolution, dynamism and motion content of the video stream, therefore the objective video quality performance of different video sequences is expected to be variable, while considering similar communication setup. As the human visual system is more sensitive to the luminance component, therefore in sampling format 


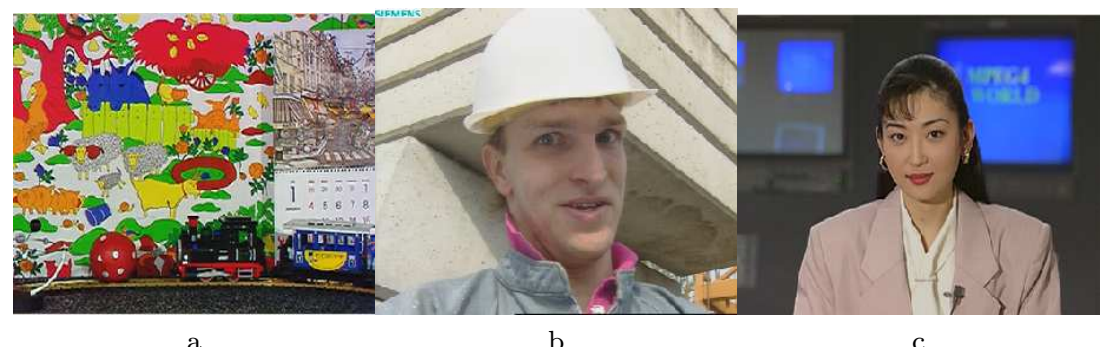

Figure 6: Video Sequences (a) MOBILE; (b) FOREMAN; (c) AKIYO.

YUV(4:2:0), more number of bits are assigned to luminance as compare to chrominance components of a video stream. The number of bits per frame for luminance and chrominance in different sampling format and video sequences are calculated from [1] as depicted in table 3. The duration of each video comprising of 45 frames at the frame rate of $15 \mathrm{fps}$ is considered. The macro-blocks in H.264/AVC are processed in the raster scan order of a group of MBs called a slice, which represents a region of a given picture that can be processed independently of each other. In our experimental setup each slice consists of $11 \mathrm{Mbs}$. The considered slice types are intra (I), inter (P) slices. All MBs in I slice are encoded using intra mode where as in $\mathrm{P}$ all MBs are coded using Intra coded mode with reference to previous frame. The encoder H.264.AVC is set at 15 fps. The resultant video frame sequence followed the pattern $I_{1} P_{2} P_{3} P_{4} \ldots P_{14}$ in which each 15 th frame is I and the predecessor 14 are p frames. All the simulation parameters are listed in Table 1 and 4.

In order to reduce the computational complexity, iterations between RSC and SBSD decoders are set to 3 and 5 for the OCM rate- 1 and $\frac{3}{4}$ respectively. The averaged results are obtained by repeating every 45 frames 160 times. The proposed system performance is exploited while considering the same overall code rate for the input H.264 encoded bit-stream.

The measurement of video quality is a major task in a scenario where there is a transmission of a compressed video over noisy channels. Subjective [34] and objective [35] techniques are widely used for measuring video quality. Human participants are required in subjective technique to assess the streaming quality and therefore it is a time-consuming solution. On the other hand PSNR is an objective metric for measuring the video quality, when the video contents, codec and underlying communication setup remains unchanged [36].

PSNR is the ratio between the signal of the power of the original signal to that of the corrupted signal. PSNR is traditionally used as a quality metric for evaluating algorithms in multimedia streaming systems. It is generally expressed in term of Mean Square Error (MSE). The MSE of an image X having dimension of size $M \times N$ and its corrupted image $\mathrm{Y}$ can be expressed by the following equation.

$$
M S E=\frac{1}{M * N} \sum_{i=1}^{M} \sum_{j=1}^{N}[X(i, j)-Y(i, j)]
$$




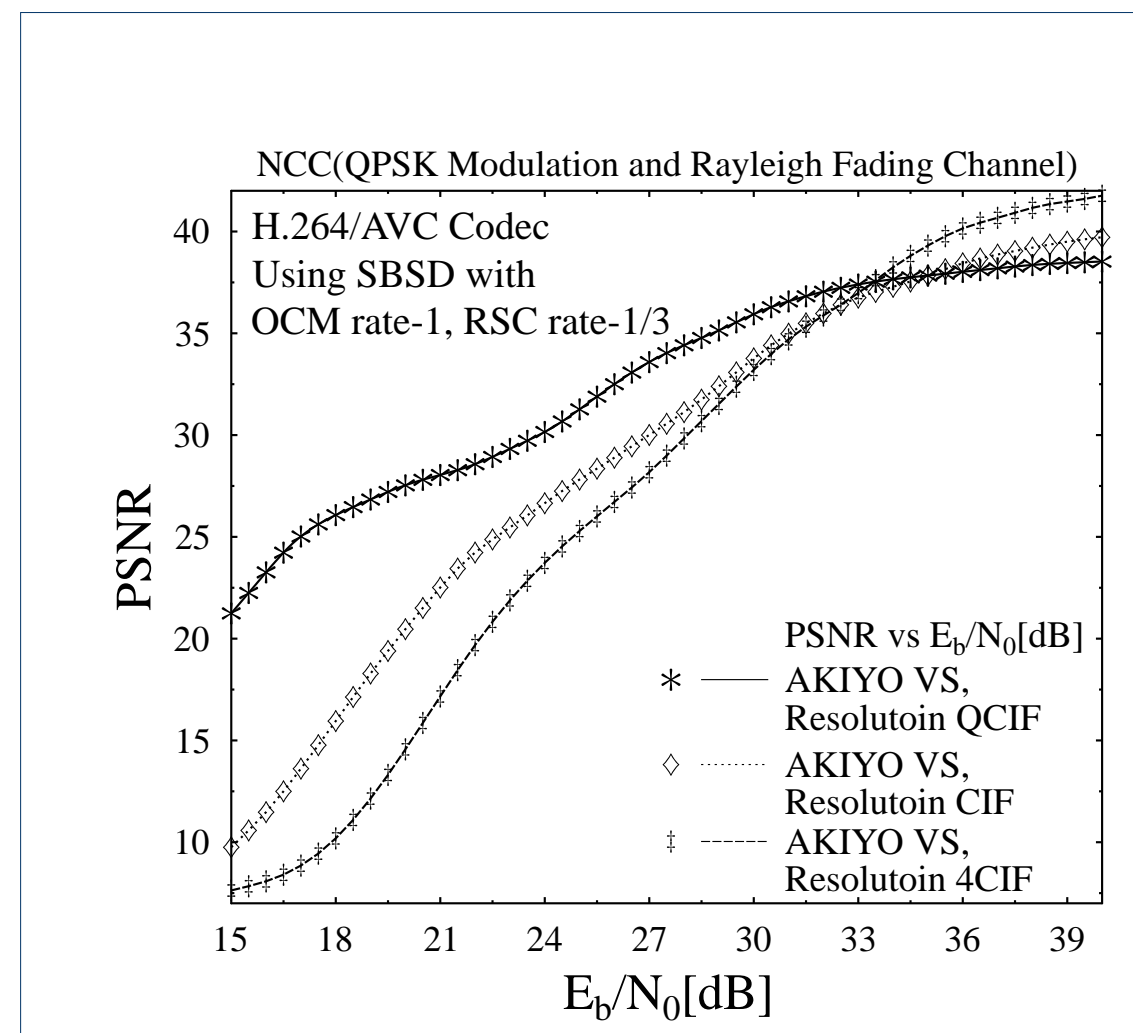

Figure 7: PSNR-Y Vs $E_{b} / N_{0}$ for NCC, AKIYO Video type.

$$
\operatorname{PSNR}(d B)=10 \cdot \log _{10} \frac{M A X_{X}}{M S E}
$$

Where $M A X_{X}$ is the maximum value of a pixel of image $\mathrm{X}$ and can be calculated as $2^{B}-1$ for a $\mathrm{B}$ bits per sample.

The BER and PSNR performance of the simulation work were plotted against varying $E_{b} / N_{0}$ values, while utilizing diverse video sequences having different resolution, motion and dynamism while employing rayleigh fading channel as a communication medium.

In our simulation scenarios OCM is employed as inner code, while RSC is used as outer channel code. Furthermore, in the considered NCC scheme QPSK modulation is employed, while in NCDSTS-SP and CDSTS-SP sphere packing modulation is used in combination with DSTS.

In order to analyze the impact of varying video resolution on the objective video quality performance of the transmission scheme its PSNR versus $E_{b} / N_{0}$ dB performance trends were plotted in Figure-7,9 and 11 for NCC, NCDSTS-SP and CDSTSSP schemes, respectively, while using AKIYO video sequence. In Figure-7,9 and 11 similar trend in performance variations due employment of different resolution videos is observed for all three employed schemes. More specifically, it is observed from Figure-7,9 and 11 that the presence of high channel noise the low resolution video outperforms its high resolution counterparts. 


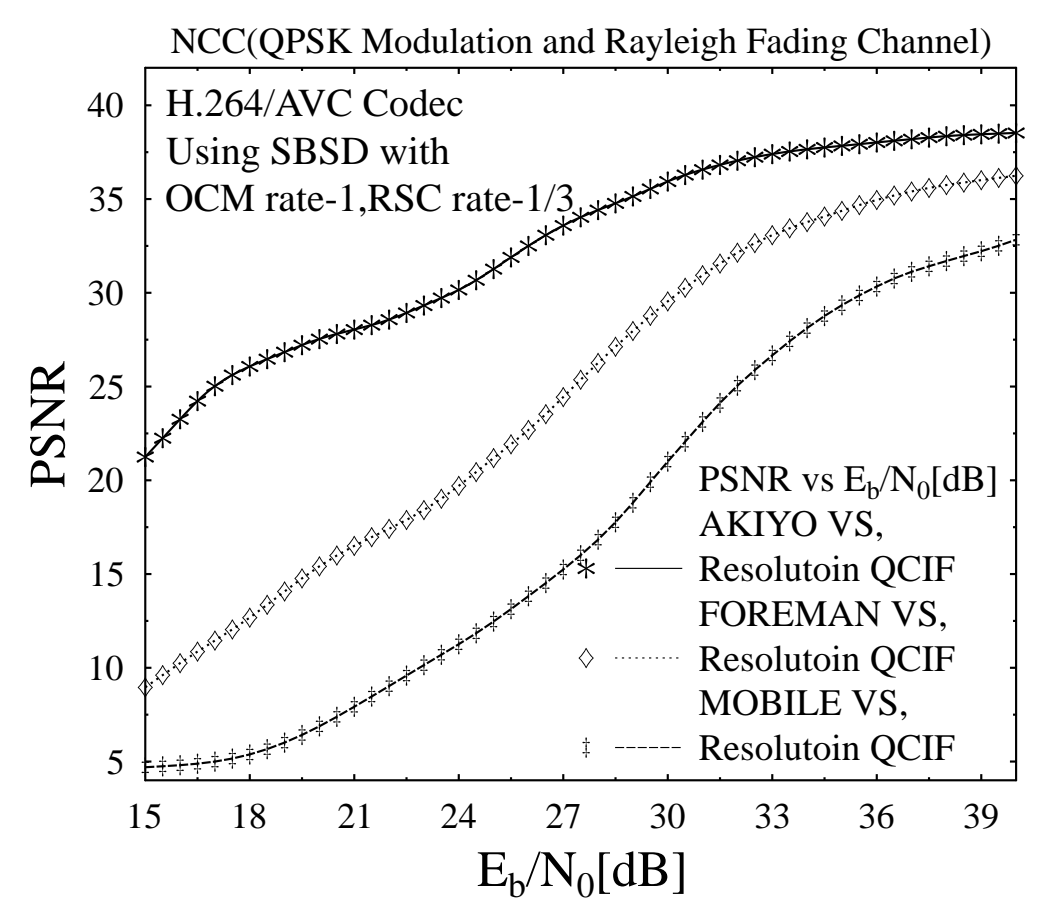

Figure 8: PSNR-Y Vs $E_{b} / N_{0}$ for NCC, AKIYO, FOREMAN and MOBILE Video Sequences.

Furthermore, in order to analyze the impact of varying motion contents and dynamism of video scene on the objective video quality performance of the transmission scheme its PSNR versus $E_{b} / N_{0}$ dB performance trends were plotted in Figure-8, 10 and 12 for NCC, NCDSTS-SP and CDSTS-SP schemes, respectively, while using AKIYO, FOREMAN and MOBILE video sequences in its QCIF video resolution.

In Figure-8, 10 and 12 similar trend in performance variations due to employment of different video sequences, with varying motion contents and dynamism, is observed for all three employed schemes. More specifically, it is observed from Figure-8, 10 and 12 that the presence of high channel noise the AKIYO video sequence with low motion contents and dynamism outperforms the FOREMAN and MOBILE video sequence with high motion contents and dynamism. This is due to the fact that in the presence of low motion contents and dynamism of video the H.264/AVC can compress the corresponding video sequence, more robustly, within the allocated bit-rate budget. Furthermore, the performance of the H.264/AVC error concealment mecahnism is more effective in video sequences with low motion contents and dynamism and as a result its objective quality performance will outperform.

The BER versus $E_{b} / N_{0} \mathrm{~dB}$ performance of the NCC, NCDSTS-SP and CDSTSSP schemes is presented in Figure-13. Its observed from Figure-13 that the BER 


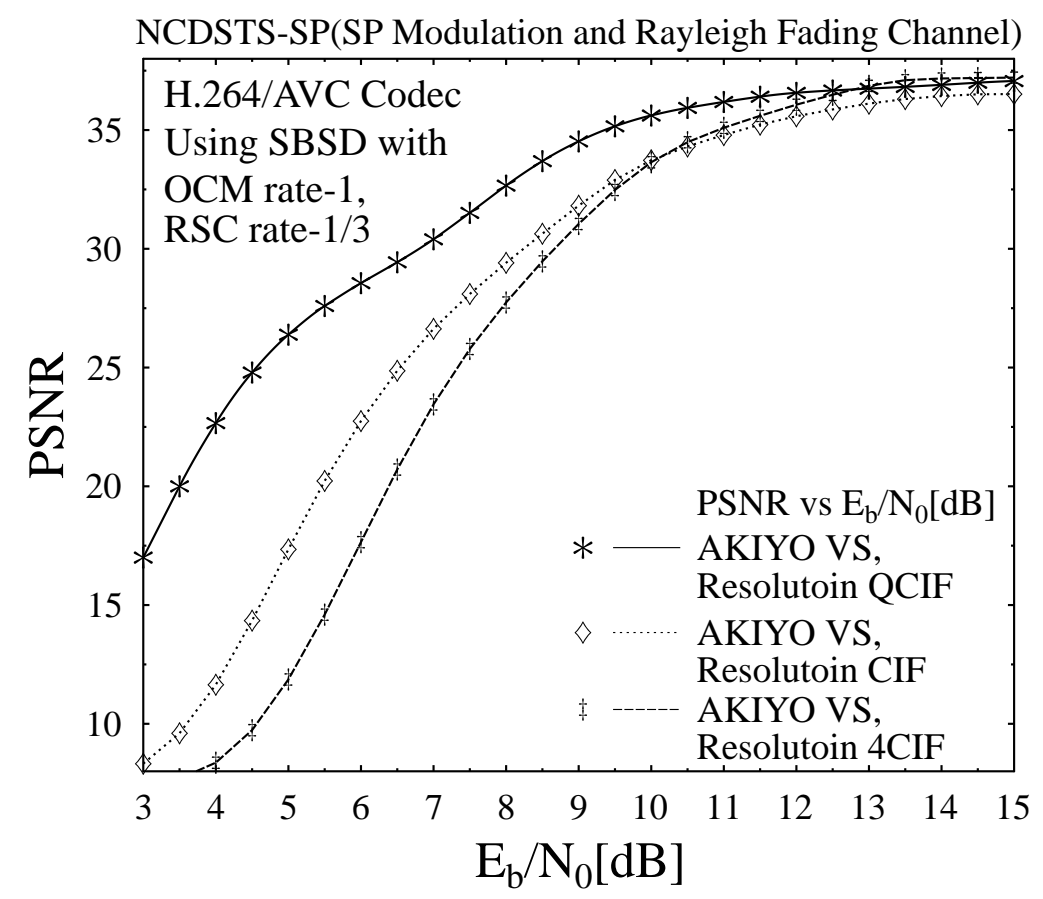

Figure 9: PSNR-Y Vs $E_{b} / N_{0}$ for NCDSTS-SP, Akiyo Video type.

performance of CDSTS-SP is best as compared to NCDSTS-SP scheme, which is performing better then NCC scheme.

Finally, its observed from the PSNR versus $E_{b} / N_{0} \mathrm{~dB}$ performance curves of the CC, NCDSTS-SP and CDSTS-SP schemes, presented in Figure-14 that the sophisticated system design of CDSTS-SP outperform its counterpart NCC, NCDSTS-SP schemes in terms of PSNR. More specifically, it is observed from Figure-14 that NCDSTS-SP results in PSNR gain of $6 \mathrm{~dB}$ and CDSTS-SP results in PSNR gain of $28 \mathrm{~dB}$ for $E_{b} / N_{0}$ value of $10 \mathrm{~dB}$, with reference to bench marker system design of NCC. 


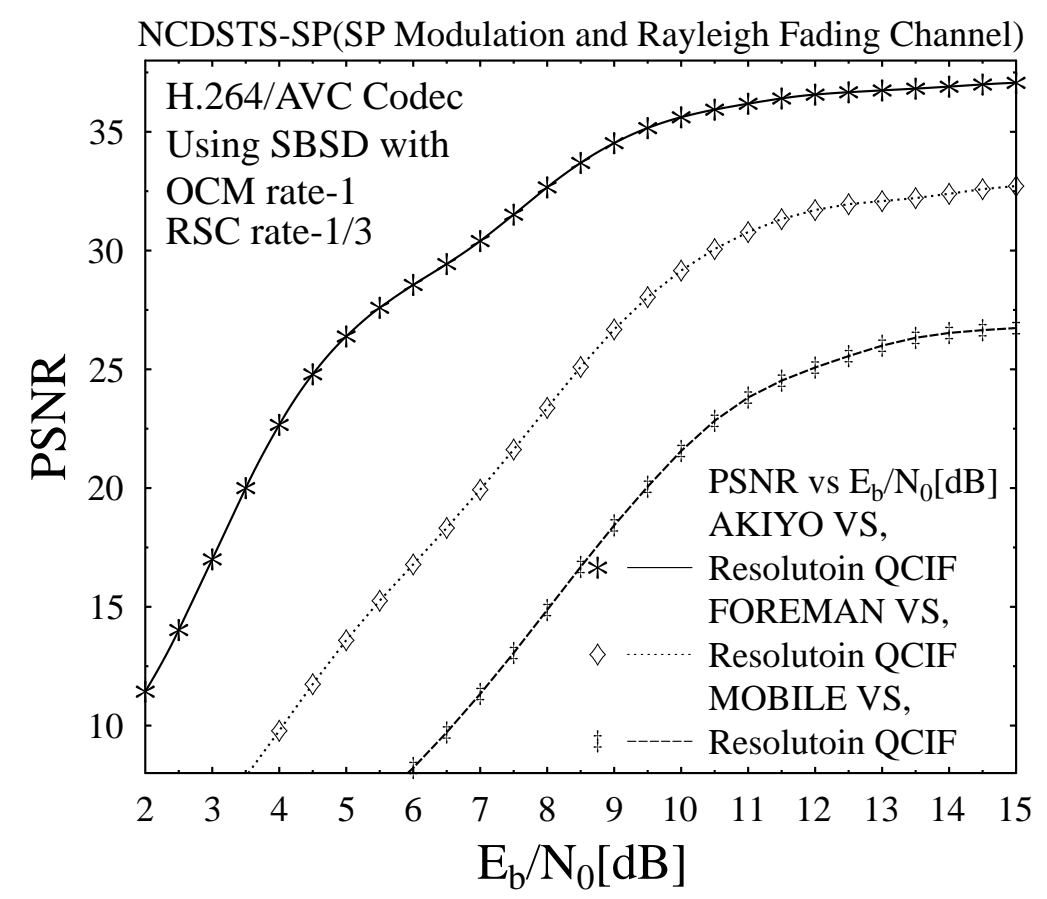

Figure 10: PSNR-Y Vs $E_{b} / N_{0}$ for NCDSTS-SP, Akiyo, Foreman and Mobile Video Sequences. 
CDSTS-SP(Sphere Packing Modulation and Rayleigh Fading Channel)

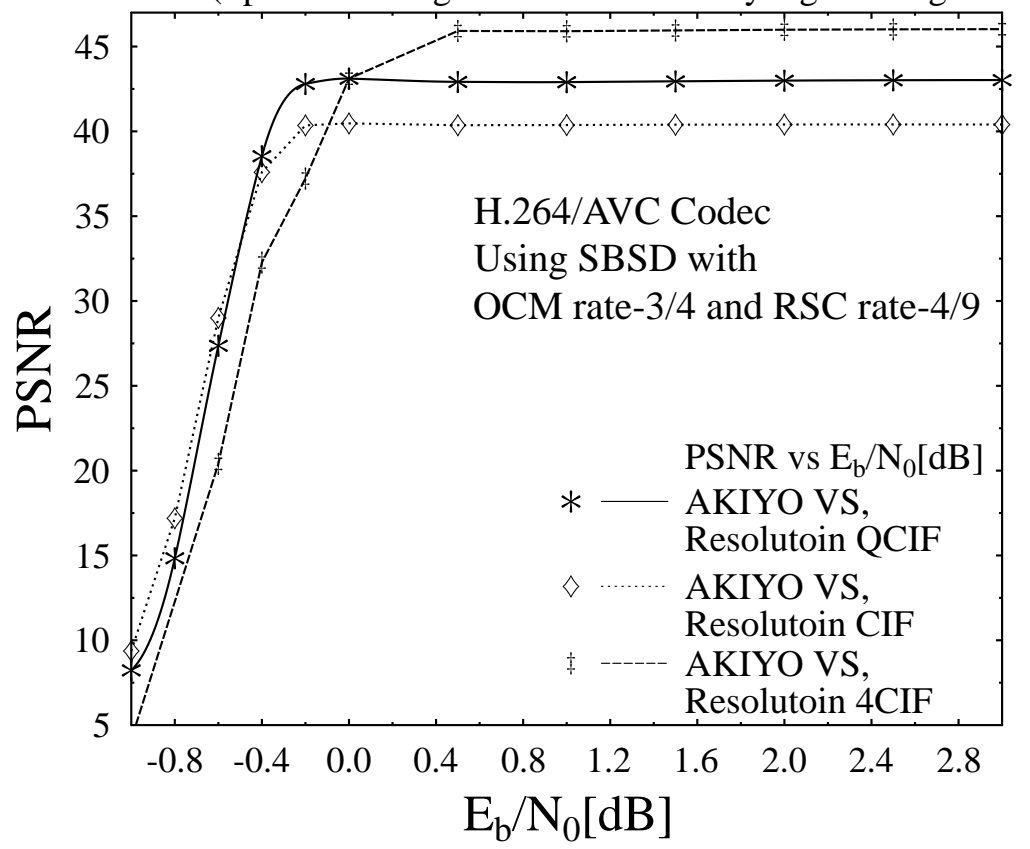

Figure 11: PSNR-Y Vs $E_{b} / N_{0}$ for CDSTS-SP, AKIYO Video type.

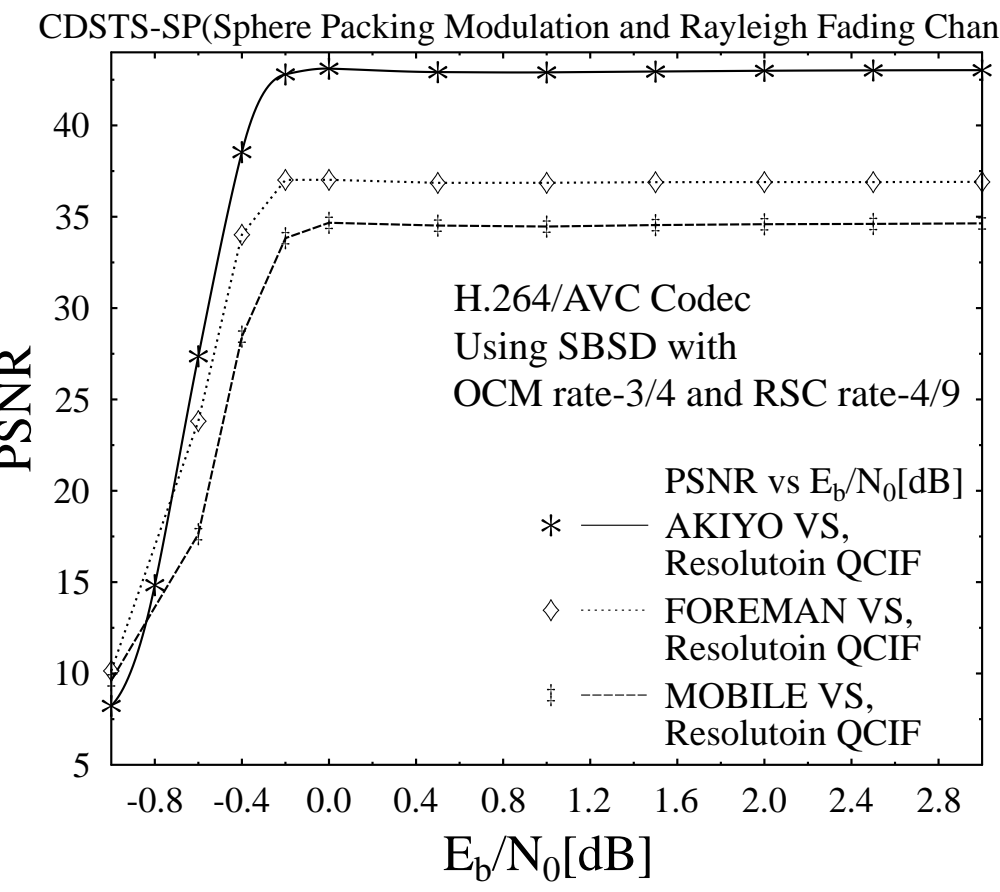

Figure 12: PSNR-Y Vs $E_{b} / N_{0}$ for CDSTS-SP, AKIYO, FOREMAN and MOBILE Video Sequences. 


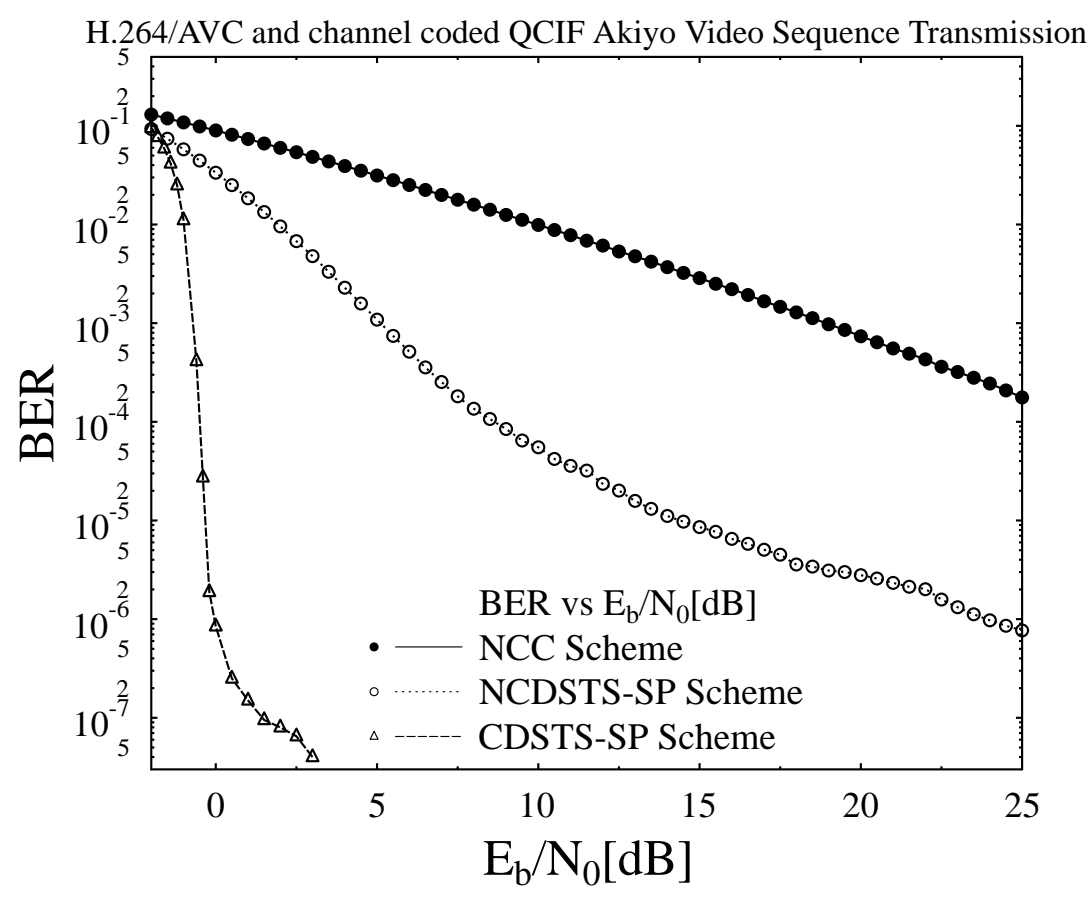

Figure 13: BER Vs $E_{b} / N_{0}$ for NCC, NCDSTS-SP and CDSTS-SP.

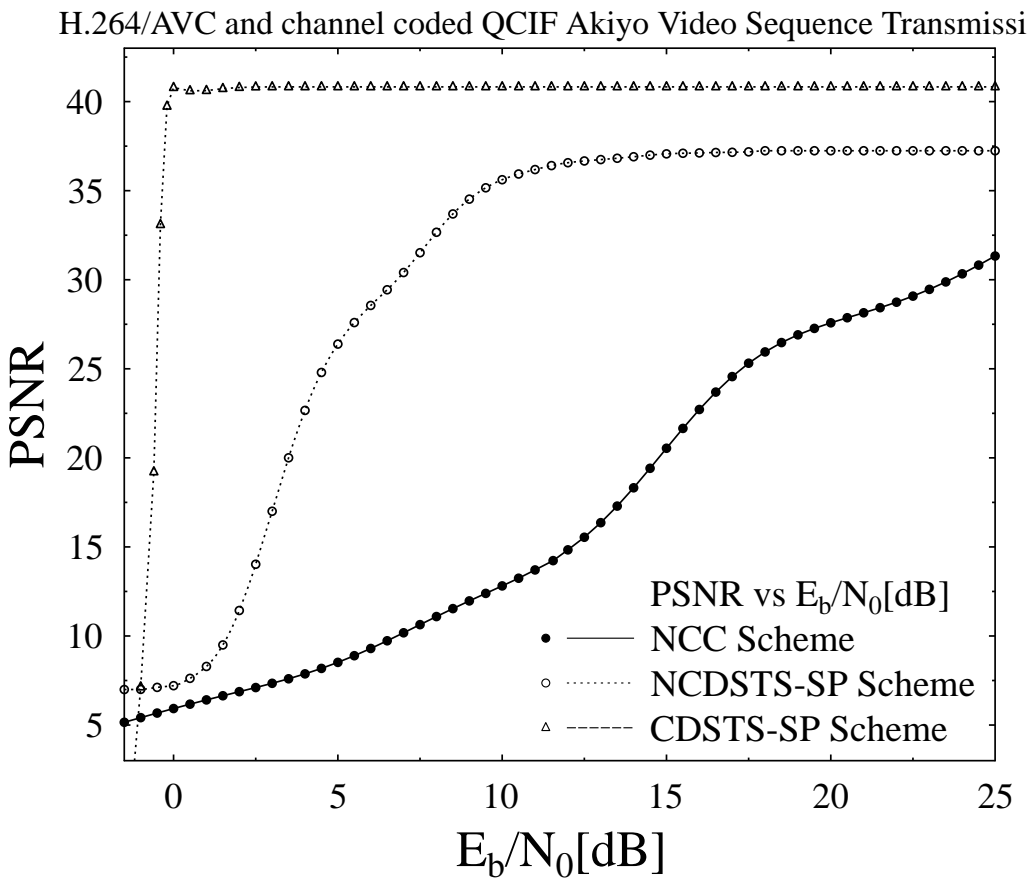

Figure 14: PSNR Vs $E_{b} / N_{0}$ for NCC, NCDSTS-SP and CDSTS-SP. 


\section{Conclusions}

This paper presents performance analysis of sophisticated channel coding and transmission schemes for reliable transmission of H.264/AVC compressed video. Three different coding and transmission schemes were presented, namely NonConvergent Coding (NCC), Non-Convergent Coding assisted with Differential Space Time Spreading (DSTS) and Sphere Packing (SP) modulation (NCDSTS-SP) and Convergent Coding assisted with Differential Space Time Spreading (DSTS) and Sphere Packing (SP) modulation (CDSTS-SP). Different diverse combination of source mapping technique (Over Complete Mapping) and channel coding technique (Recursive System Convolution) were employed and their convergence behavior is analyzed with the aid of Extrinsic Information Transfer (EXIT) Chart. Furthermore, to improve the diversity gain of the transceiver advanced Sphere Packing (SP) modulation technique assisted by Differential Space Time Spreading (DSTS) is employed. The BER and PSNR performance of the proposed schemes were plotted against varying $E_{b} / N_{0}$ values, while utilizing diverse video sequences having different resolution, motion and dynamism while employing Rayleigh fading channel as a communication medium. It was observed that the presence of high channel noise the low resolution videos outperforms its high resolution counterparts. Furthermore, it was observed that in the presence of high channel noise, the AKIYO video sequence with low motion contents and dynamism outperforms the FOREMAN and MOBILE video sequence with high motion contents and dynamism. Furthermore, it is observed from that the BER and PSNR versus $E_{b} / N_{0} \mathrm{~dB}$ performance of the NCC, NCDSTS-SP and CDSTS-SP schemes that the BER performance of CDSTS-SP is best as compared to NCDSTS-SP scheme, which is performing better then NCC scheme. More specifically, it is observed that the NCDSTS-SP results in PSNR gain of $6 \mathrm{~dB}$ and CDSTS-SP results in PSNR gain of $28 \mathrm{~dB}$ for $E_{b} / N_{0}$ value of $10 \mathrm{~dB}$, with reference to bench marker system design of NCC. As a future work, our focusing will be on the performance analysis of H.265/HEVC, while considering the developed coding, modulation and transmission schemes.

Competing interests

The authors declare that they have no competing interests.

Author's contributions

Nasru Minallah and Khadem Ullah conceived the main ideas presented in this research work, performed the simulations and analyses. Imran Ullah Khan contributed to critically revise and finlize the research. All authors equally contributed in the paper writing.

Acknowledgements

This work is executed in the field of Multimedia Streaming under the project NCBC-UETP and is supported by Harbin Engineering University, Heilongjiang, China, under Grant No: HEUCFG201712/3072019CFJ0518

\section{Author details}

${ }^{1}$ Department of Computer Systems Engineering, University of Engineering and Technology Peshawar, 25000, Peshawar, Pakistan. ${ }^{2}$ National Center of Big Data and Cloud Computing, University Of Engineering and Technology Peshawar(NCBC-UETP), 25000 Peshawar, Pakistan. ${ }^{3}$ College of Underwater Acoustics Engineering, Harbin Engineering University Heilongjiang, 150001 Harbin, China.

References

1. Richardson, I.E.: H. 264 and MPEG-4 Video Compression: Video Coding for Next-generation Multimedia. John Wiley \& Sons, ??? (2004)

2. Shannon, C.E.: A mathematical theory of communication. Bell System Technical Journal 27(3), 379-423 (1948). doi:10.1002/j.1538-7305.1948.tb01338.x

3. Hamming, R.W.: Error detecting and error correcting codes. Bell System Technical Journal 29(2), 147-160 (1950). doi:10.1002/j.1538-7305.1950.tb00463.x 
4. Elias, P.: Coding for noisy channels. Technical report, IRE Convention Record, pt.4, pp. 37-47, (1955)

5. Hagelbarger, D.W.: Recurrent codes: Easily mechanized, burst-correcting, binary codes. Bell System Technical Journal 38(4), 969-984 (1959). doi:10.1002/j.1538-7305.1959.tb01584.x

6. Wozencraft, J.M., Reiffen., B.: Sequential decoding, vol. 10. The MIT Press, (1961)

7. Fano, R.: A heuristic discussion of probabilistic decoding. IEEE Transactions on Information Theory $9(2)$, 64-74 (1963). doi:10.1109/tit.1963.1057827

8. Viterbi, A.: Error bounds for convolutional codes and an asymptotically optimum decoding algorithm. IEEE Transactions on Information Theory 13(2), 260-269 (1967). doi:10.1109/tit.1967.1054010

9. Bahl, L., Cocke, J., Jelinek, F., Raviv, J.: Optimal decoding of linear codes for minimizing symbol error rate (corresp.). IEEE Transactions on Information Theory 20(2), 284-287 (1974). doi:10.1109/tit.1974.1055186

10. Berrou, C., Glavieux, A., Thitimajshima, P.: Near shannon limit error-correcting coding and decoding: Turbo-codes. 1. In: Proceedings of ICC 93 - IEEE International Conference on Communications. IEEE. doi:10.1109/icc.1993.397441

11. Berrou, C., Glavieux., A.: Near optimum error correcting coding and decoding: Turbo-codes. The best of the best: fifty years of communications and networking research45 (2007)

12. Steele, R., Hanzo., L.: Mobile radio communications: Second and third generation cellular and watm systems:. 2nd. IEEE Press-John Wiley, (1999)

13. Hagenauer, J., Hoeher, P.: A viterbi algorithm with soft-decision outputs and its applications. In: IEEE Global Telecommunications Conference, 1989, and Exhibitio. Communications Technology for the 1990s and Beyond. IEEE. doi:10.1109/glocom.1989.64230

14. Koch, W., Baier, A.: Optimum and sub-optimum detection of coded data disturbed by time-varying intersymbol interference (applicable to digital mobile radio receivers). In: [Proceedings] GLOBECOM 90: IEEE Global Telecommunications Conference and Exhibition. IEEE. doi:10.1109/glocom.1990.116774

15. Chen, C., Wang, L., Lau, F.C.: Joint optimization of protograph Idpc code pair for joint source and channel coding. IEEE Transactions on Communications 66(8), 3255-3267 (2018)

16. El-Hajjar, M.H.: Near-capacity mimos using iterative detection. PhD diss., University of Southampton, (2008)

17. Telatar, E.: Capacity of multi-antenna gaussian channels. European Transactions on Telecommunications 10(6), 585-595 (1999). doi:10.1002/ett.4460100604

18. Foschini, G.J., Chizhik, D., Gans, M.J., Papadias, C., Valenzuela, R.A.: Analysis and performance of some basic space-time architectures. IEEE Journal on Selected Areas in Communications 21(3), 303-320 (2003). doi:10.1109/jsac.2003.809456

19. Alamouti, S.M.: A simple transmit diversity technique for wireless communications. (1998 (1998).)

20. Tarokh, V., Jafarkhani, H.: A differential detection scheme for transmit diversity. IEEE journal on selected areas in communications 18(7), 1169-1174 (2000)

21. El-Hajjar, M., Alamri, O., Ng, S.X., Hanzo, L.: Turbo detection of precoded sphere packing modulation using four transmit antennas for differential space-time spreading. IEEE Transactions on Wireless Communications 7(3), 943-952 (2008). doi:10.1109/twc.2008.060708

22. Stockhammer, T., Hannuksela, M.M., Wiegand, T.: H.264/AVC in wireless environments. IEEE Transactions on Circuits and Systems for Video Technology 13(7), 657-673 (2003). doi:10.1109/tcsvt.2003.815167

23. Ghandi, M.M., Barmada, B., Jones, E.V., Ghanbari, M.: H.264 layered coded video over wireless networks: Channel coding and modulation constraints. EURASIP Journal on Advances in Signal Processing 2006(1) (2006). doi:10.1155/asp/2006/85870

24. Adrat, M., Vary., P.: Iterative source-channel decoding: improved system design using exit charts. EURASIP Journal on Applied Signal Processing 2005 (2005): 928-941. (2005)

25. Hanzo, P.C. Lajos, Streit., J.: Video compression and communications: from basics to h. 261, h. 263, h. 264, mpeg4 for dvb and hsdpa-style adaptive turbo-transceivers. John Wiley \& Sons, (2007)

26. ISO/IEC 11172:: Information technology coding of moving pictures and associated audio for digital storage media at up to about $1.5 \mathrm{mbit} / \mathrm{s}$, . Geneva, (1993)

27. ISO/IEC 13818-2:: Generic coding of moving pictures and associated audio information part 2: Video, 1994. also ITU-T Recommendation H.262

28. ITU-T Recommendation H.263: Video coding for low bit rate communication,. version 1, Nov. 1995; version 2, Jan. 1998; version 3, Nov. 2000.

29. ISO/IEC 14496 2:: Information technology coding of audiovisual objects part 2: visual,. Geneva, (2000)

30. Ostermann, J., Bormans, J., List, P., Marpe, D., Narroschke, M., Pereira, F., Stockhammer, T., Wedi, T.: Video coding with h.264/AVC: tools, performance, and complexity. IEEE Circuits and Systems Magazine 4(1), 7-28 (2004). doi:10.1109/mcas.2004.1286980

31. Proakis, J.G., Salehi, M.: Digital Communications vol. 4. McGraw-hill New York, ??? (2001)

32. Bahl, L., Jelinek, F.: Rate $1 / 2$ convolutional codes with complementary generators. IEEE Transactions on Information Theory 17(6), 718-727 (1971)

33. Ten Brink, S.: Convergence behavior of iteratively decoded parallel concatenated codes. IEEE transactions on communications 49, no. 10 (2001): 1727-1737 (2001)

34. BT, R.I.-R.: Methodology for the subjective assessment of the quality of television pictures (2002)

35. Webster, A.A., Jones, C.T., Pinson, M.H., Voran, S.D., Wolf, S.: Objective video quality assessment system based on human perception. In: Human Vision, Visual Processing, and Digital Display IV, vol. 1913, pp. 15-26 (1993). International Society for Optics and Photonics

36. Huynh-Thu, Q., Ghanbari, M.: Scope of validity of psnr in image/video quality assessment,. in Electronics Letters, vol. 44 , no. 13 , pp. 800-801, (19 June 2008.) 

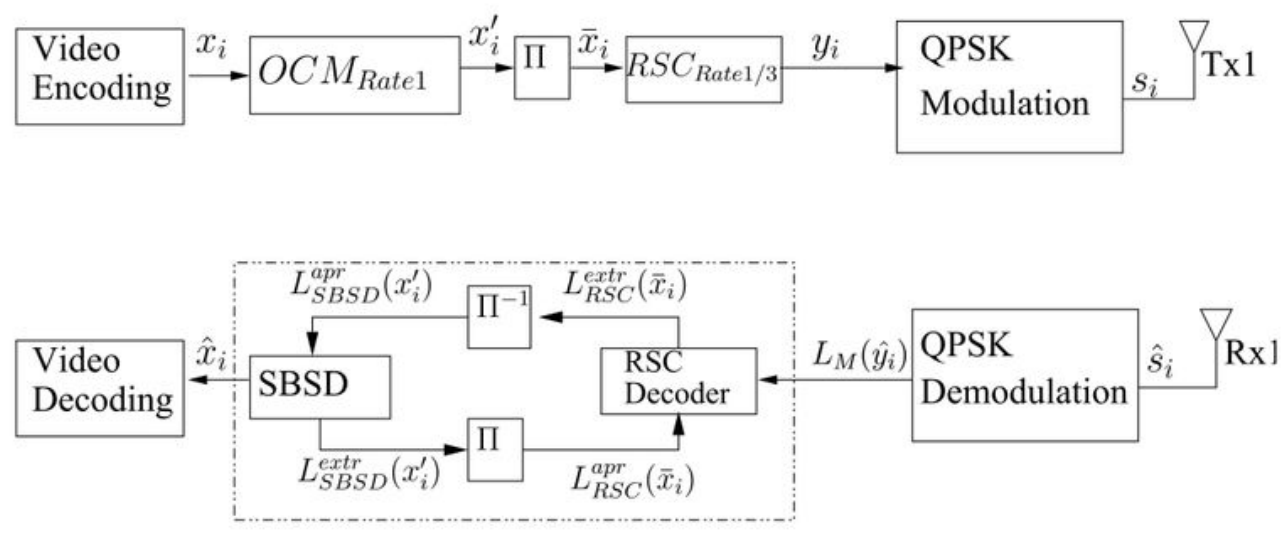

Iterative Soft Decoding

(a)
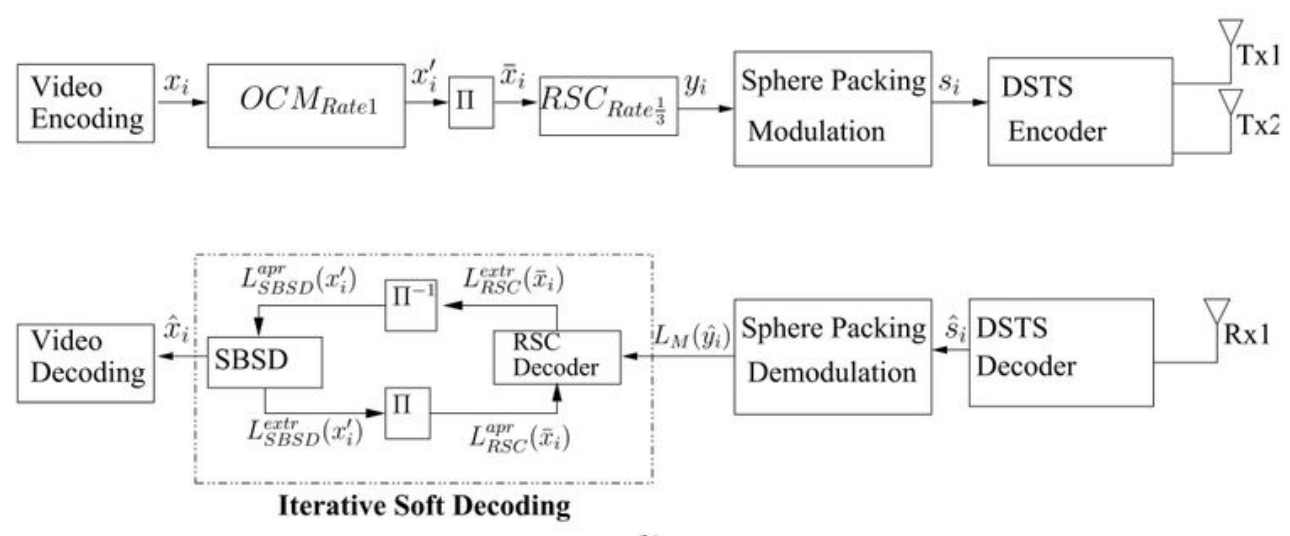

(b)
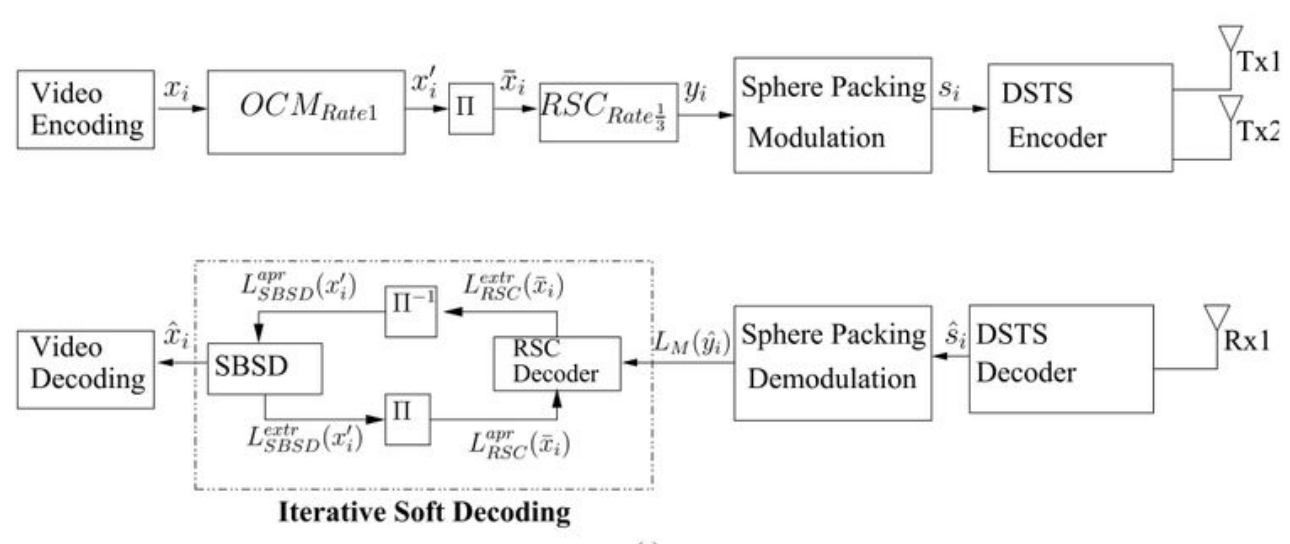

(c)

Figure 1

Proposed Systems (a) NCC; (b) NCDSTS-SP; (c) CDSTS-SP. 


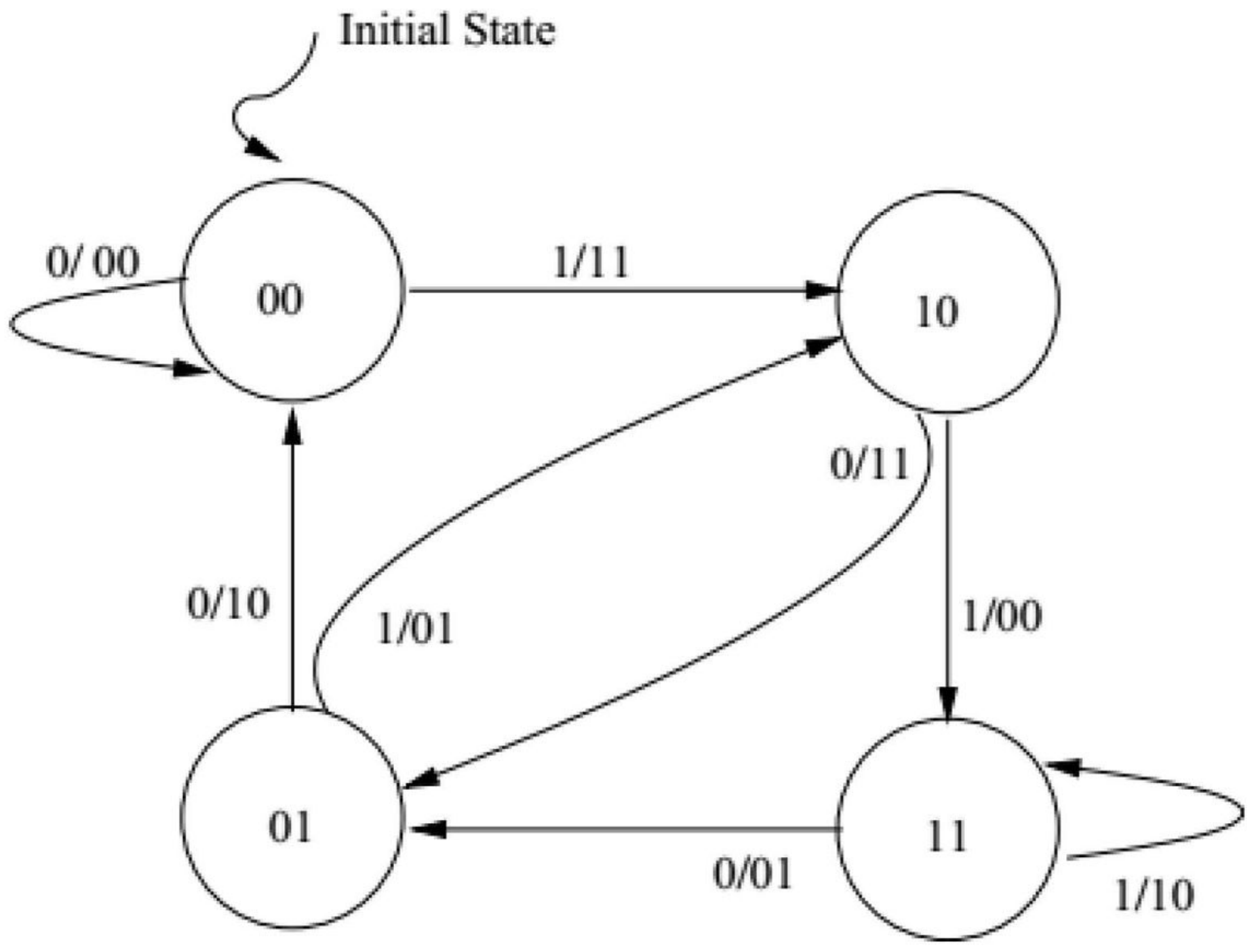

Figure 2

State Machine Diagram of Convolutional Coding 


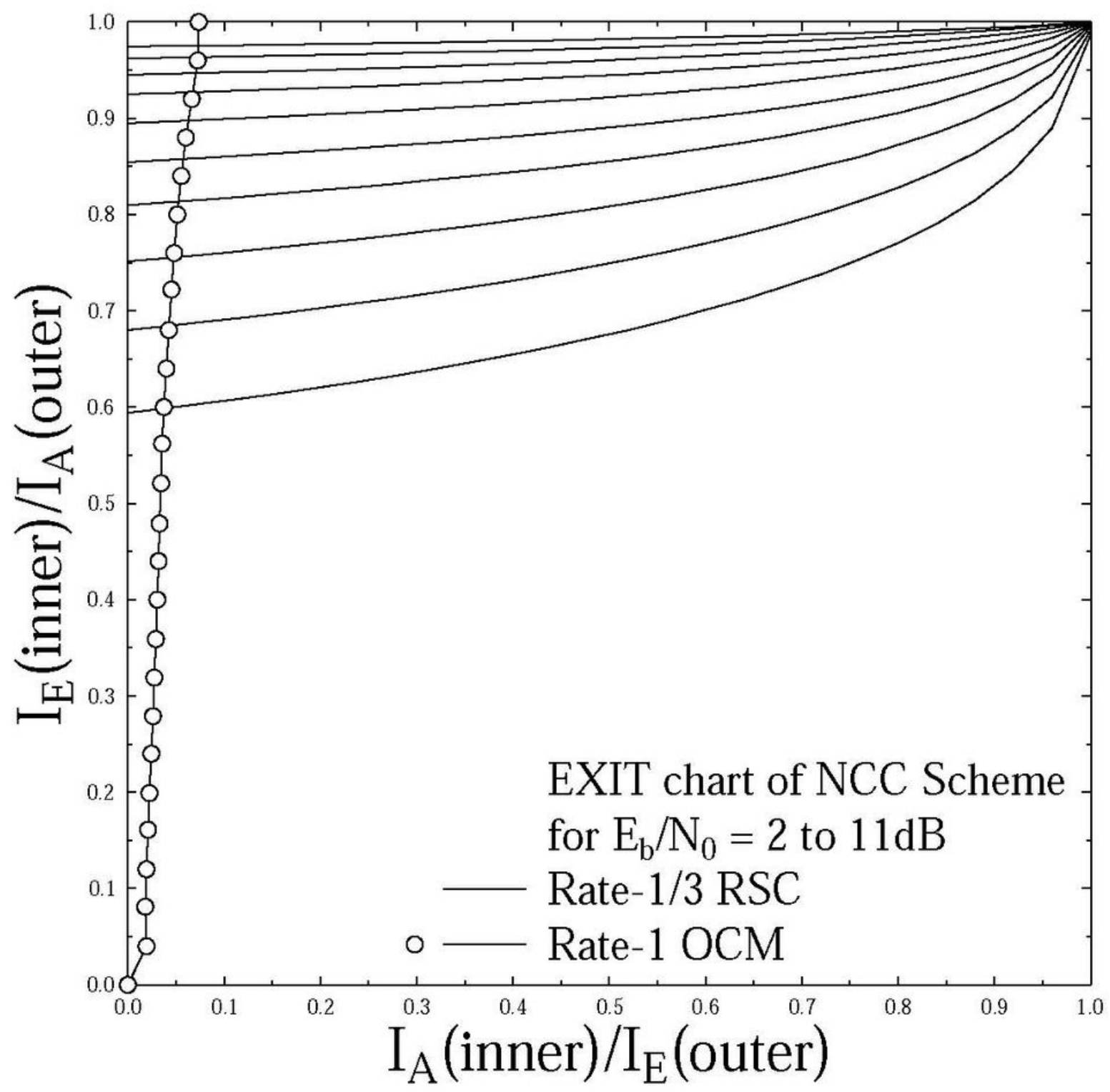

Figure 3

EXIT chart - Rate-1 OCM Outer Code and Rate-1/3 RSC Inner Code 


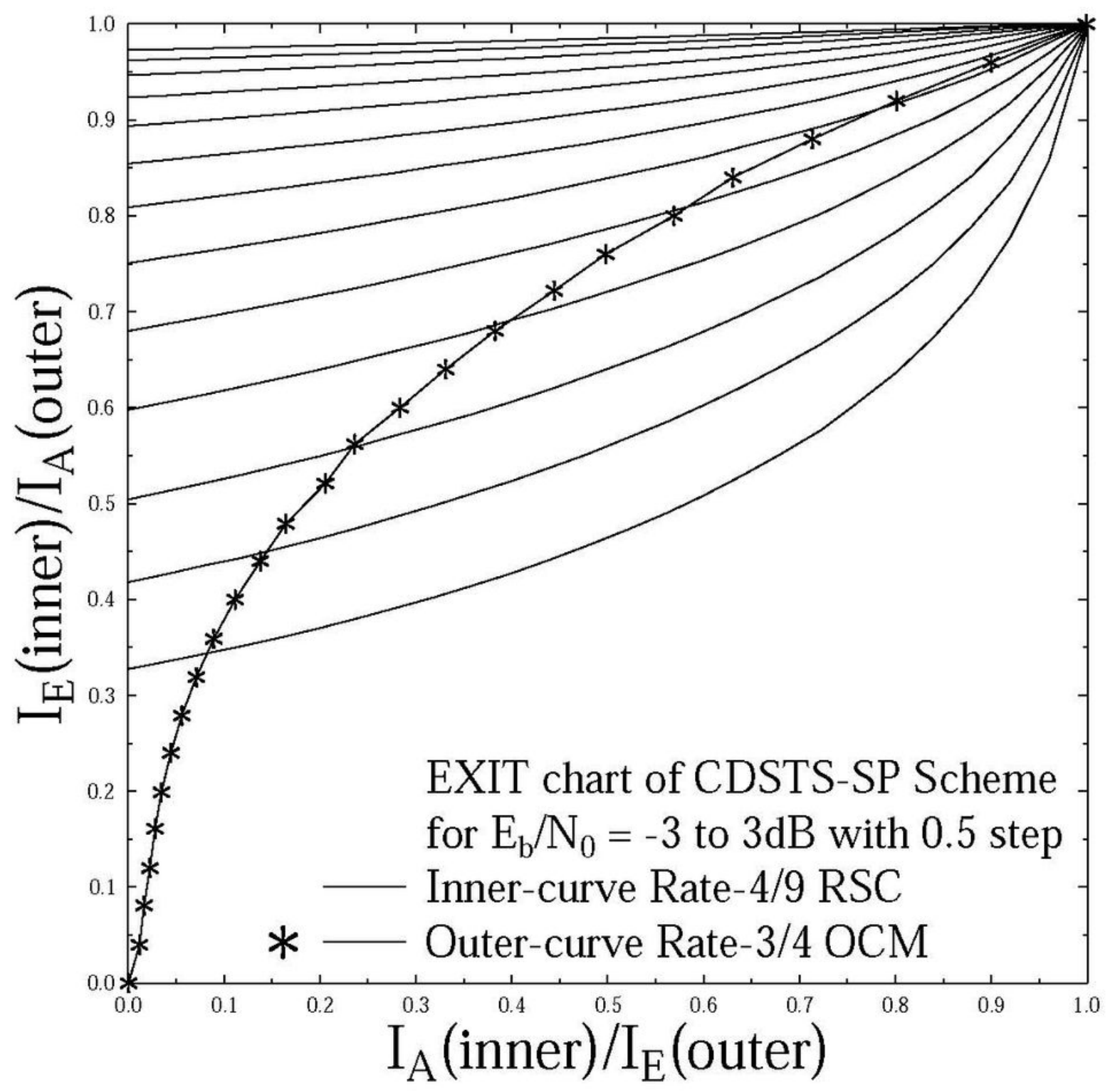

Figure 4

EXIT chart - Rate-3/4 OCM Outer Code and Rate-4/9 RSC Inner Code 


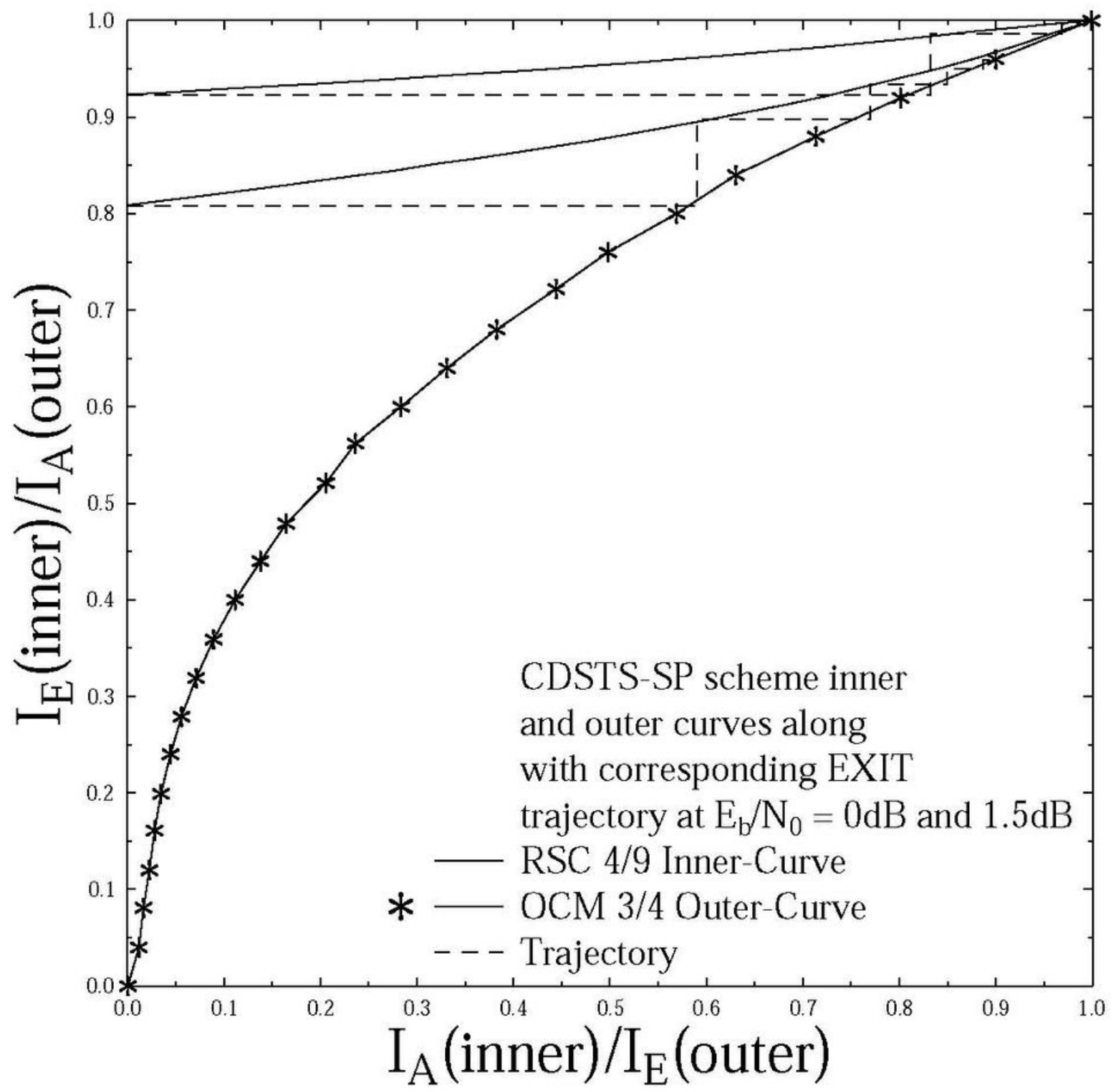

Figure 5

EXIT Trajectory of CDSTS-SP for $E b=N O=-0: 3 \mathrm{~dB}$ 


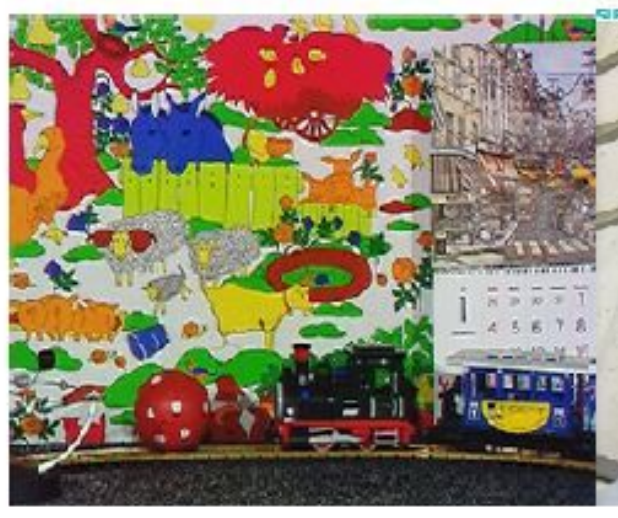

a

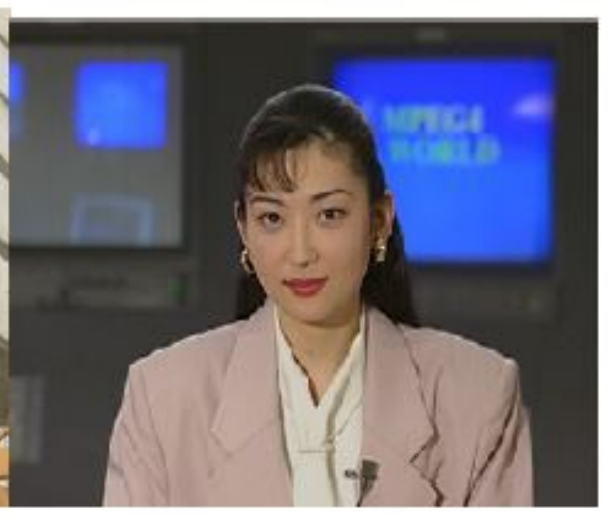

c

Figure 6

Video Sequences (a) MOBILE; (b) FOREMAN; (c) AKIYO.

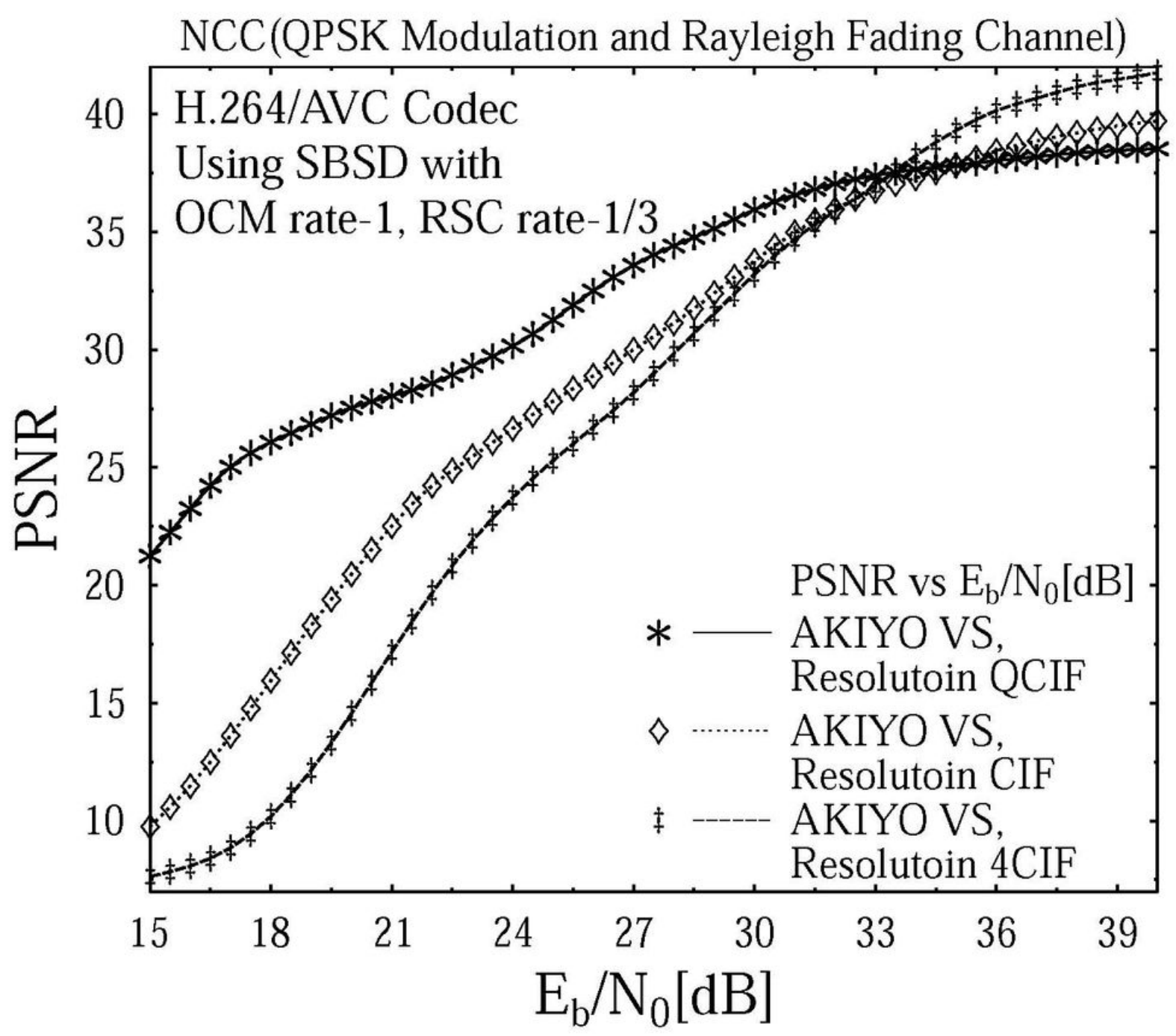


Figure 7

PSNR-Y Vs Eb=NO for NCC, AKIYO Video type.

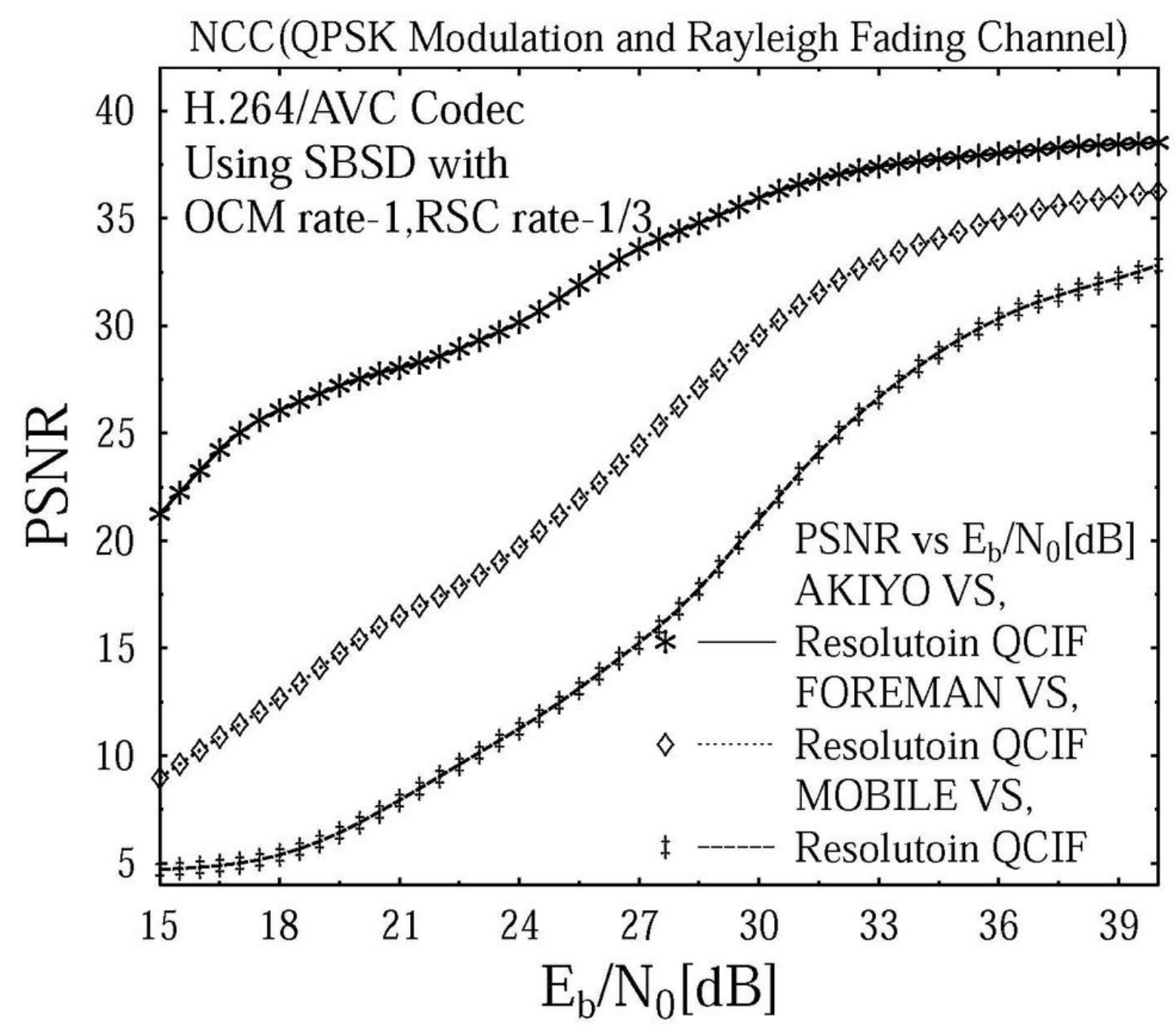

Figure 8

PSNR-Y Vs Eb=NO for NCC, AKIYO, FOREMAN and MOBILE Video Sequences. 


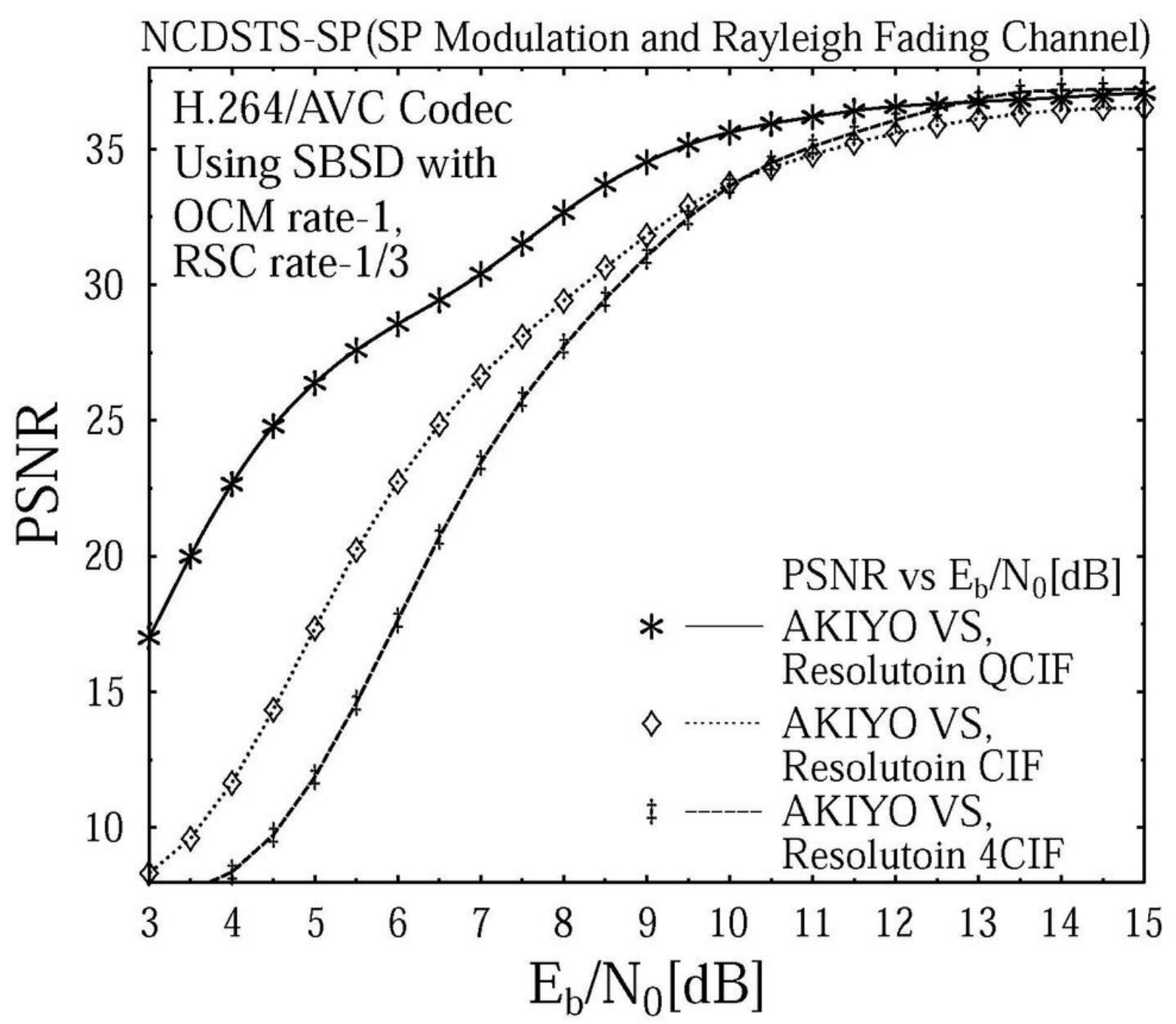

Figure 9

PSNR-Y Vs Eb=N0 for NCDSTS-SP, Akiyo Video type. 


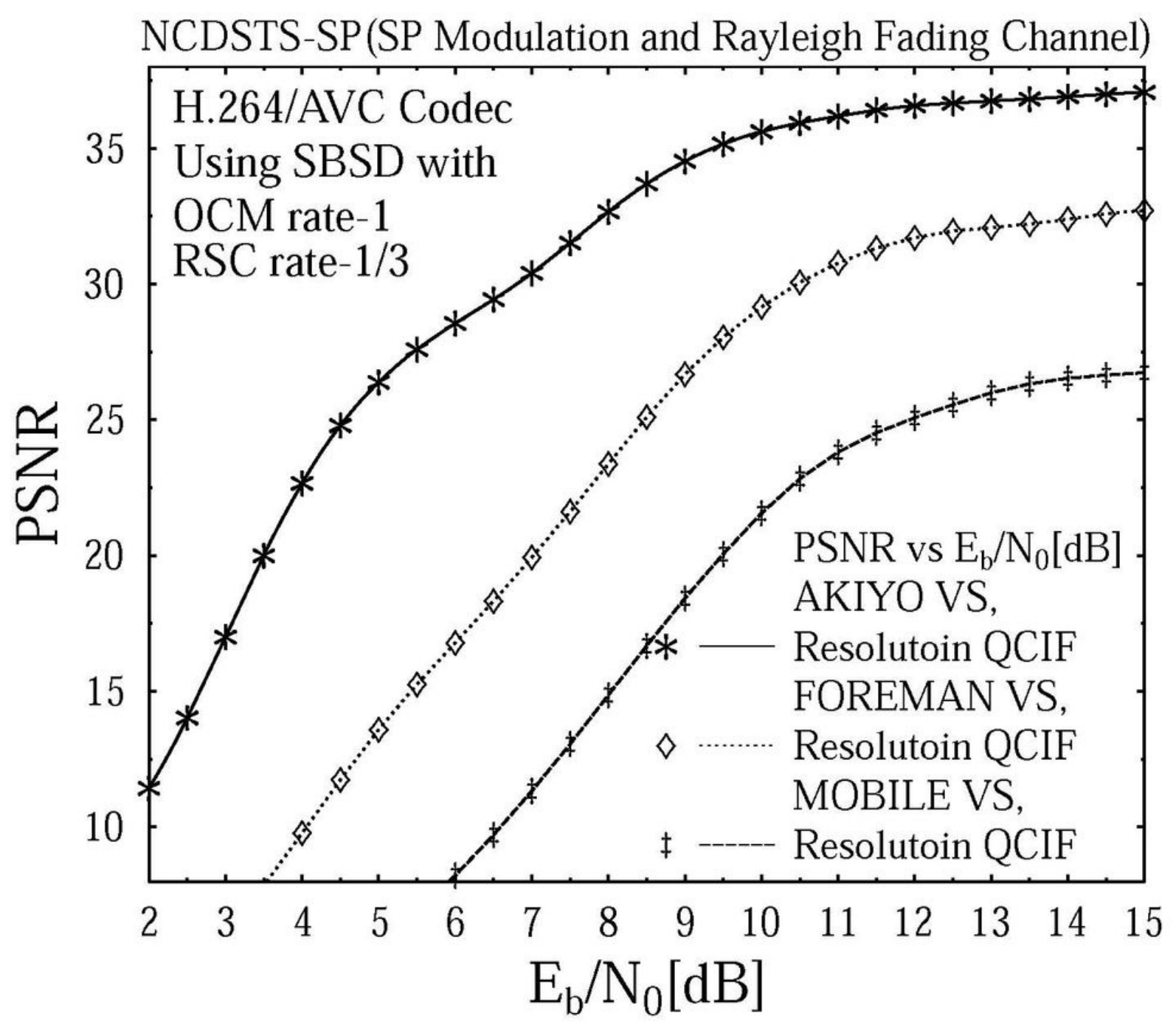

Figure 10

PSNR-Y Vs Eb=NO for NCDSTS-SP, Akiyo, Foreman and Mobile Video Sequences. 


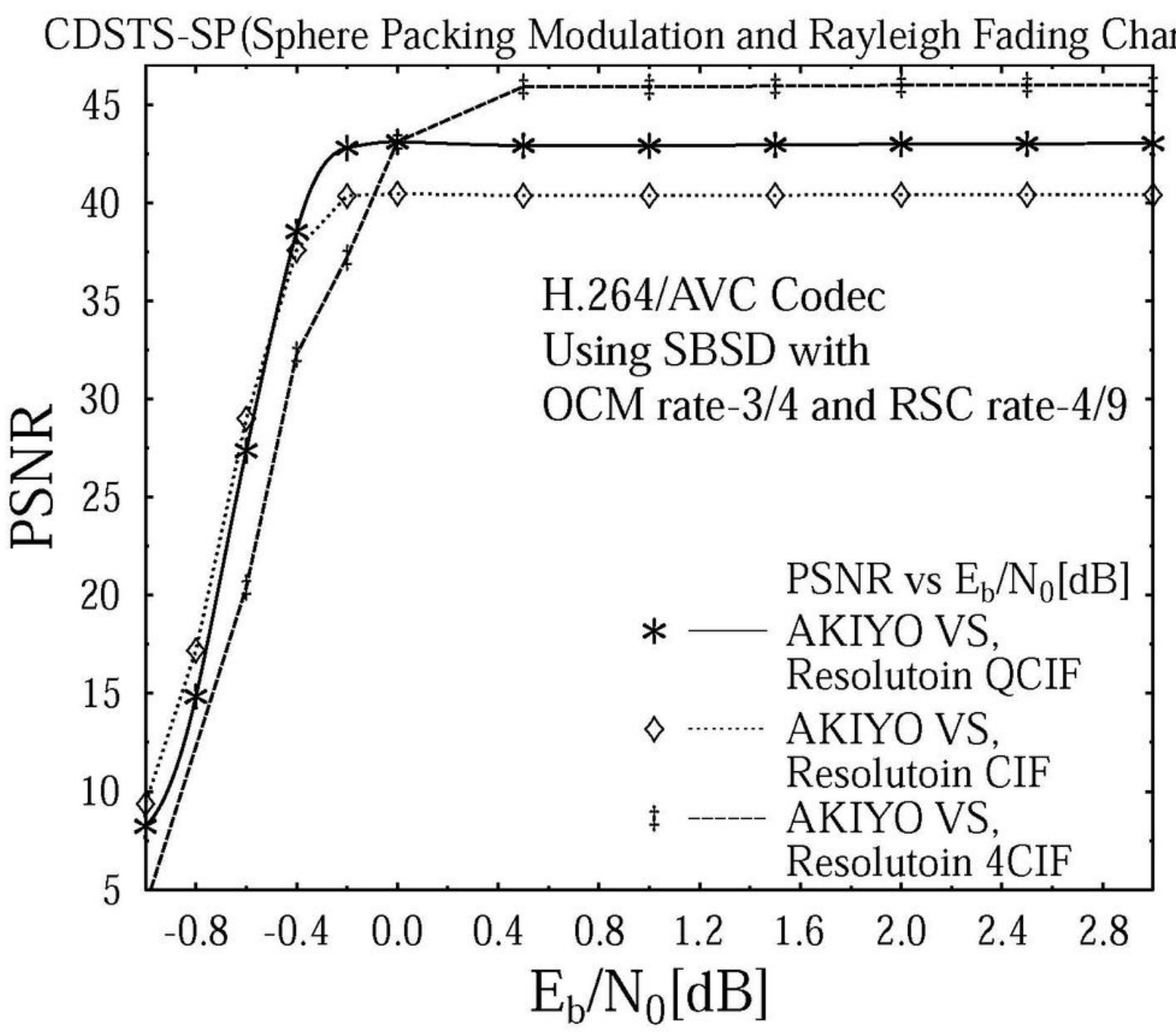

Figure 11

PSNR-Y Vs Eb=NO for CDSTS-SP, AKIYO Video type. 
CDSTS-SP(Sphere Packing Modulation and Rayleigh Fading Channel)

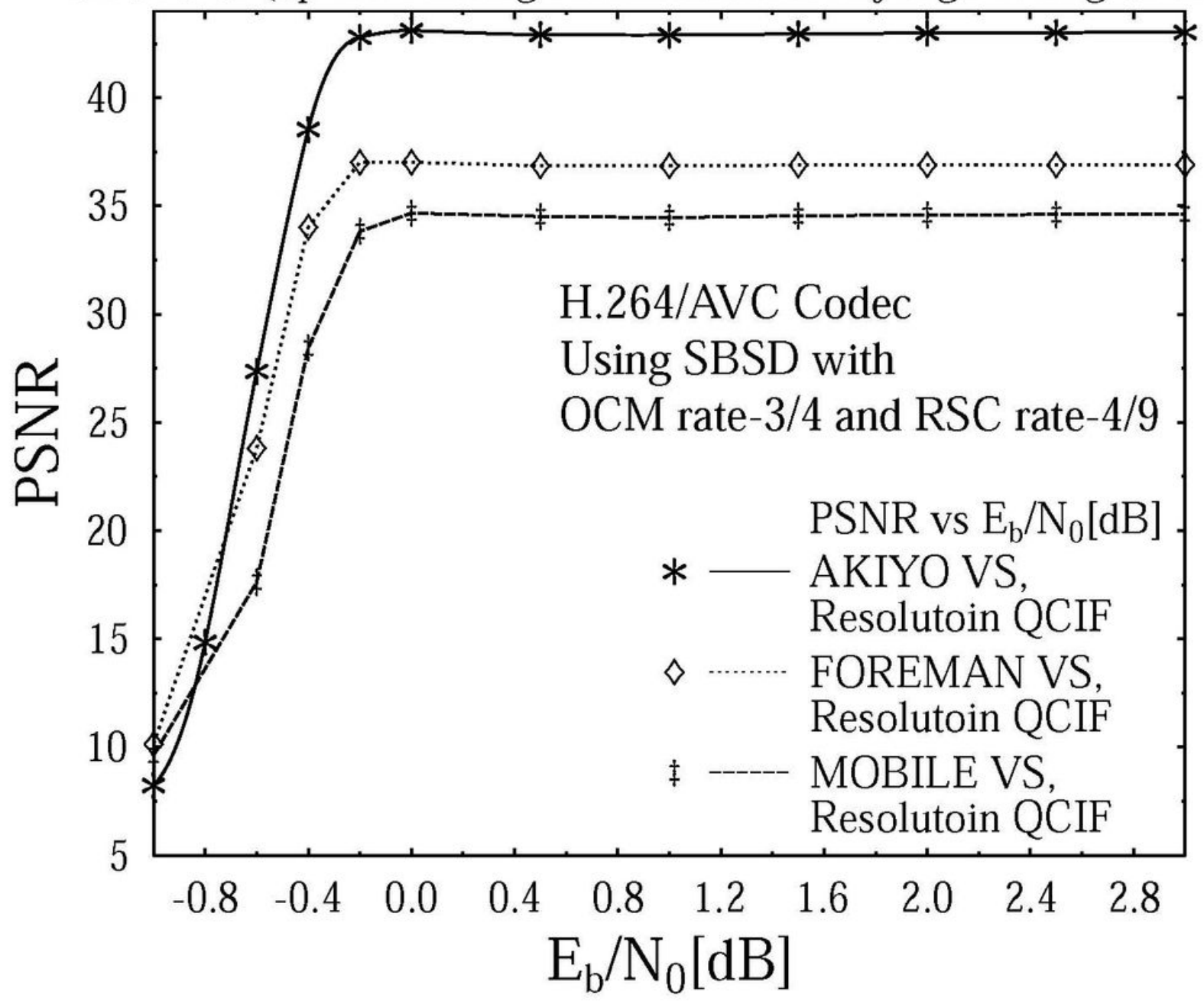

Figure 12

PSNR-Y Vs Eb=NO for CDSTS-SP, AKIYO, FOREMAN and MOBILE Video Sequences. 


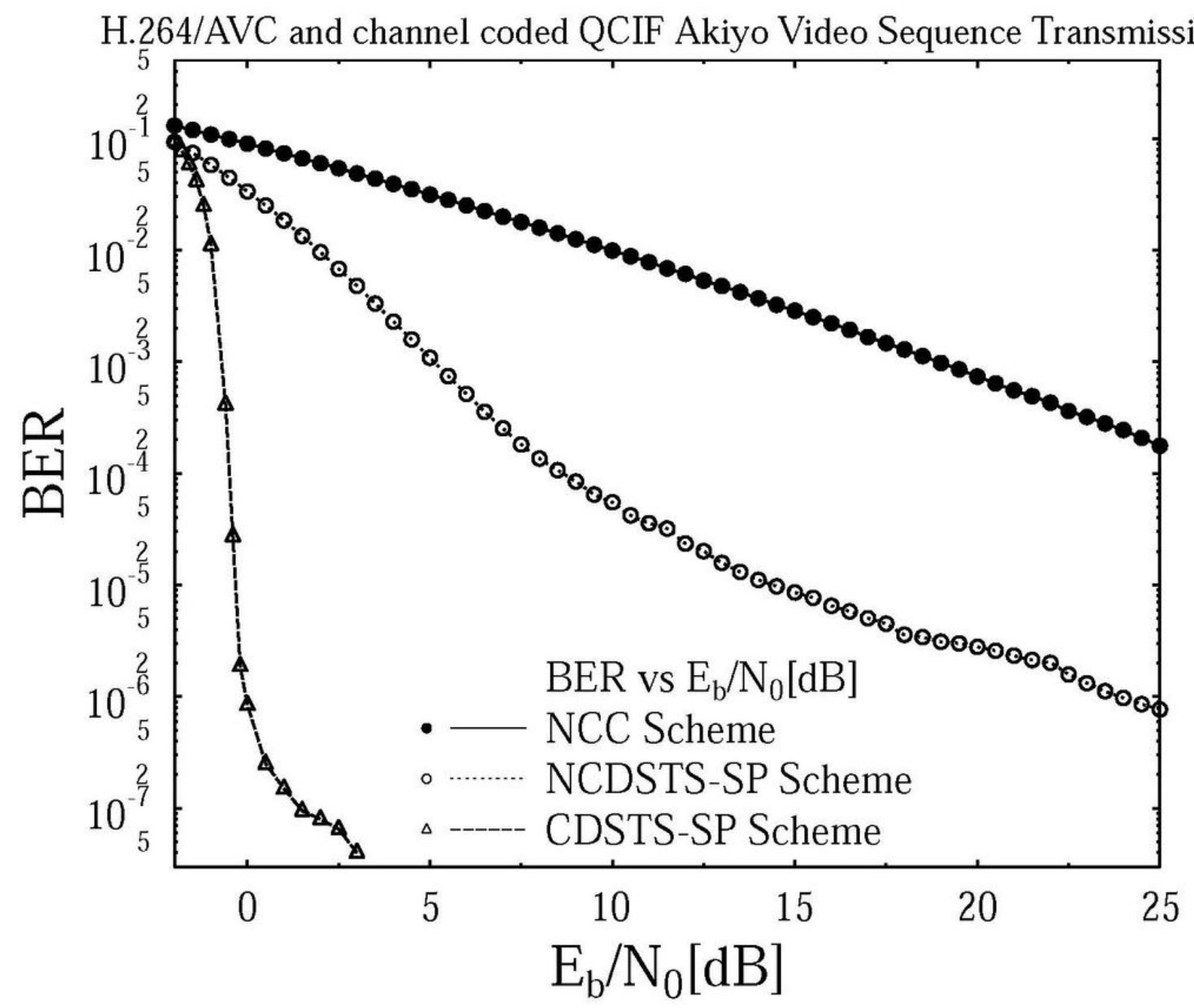

Figure 13

BER Vs Eb=NO for NCC, NCDSTS-SP and CDSTS-SP. 


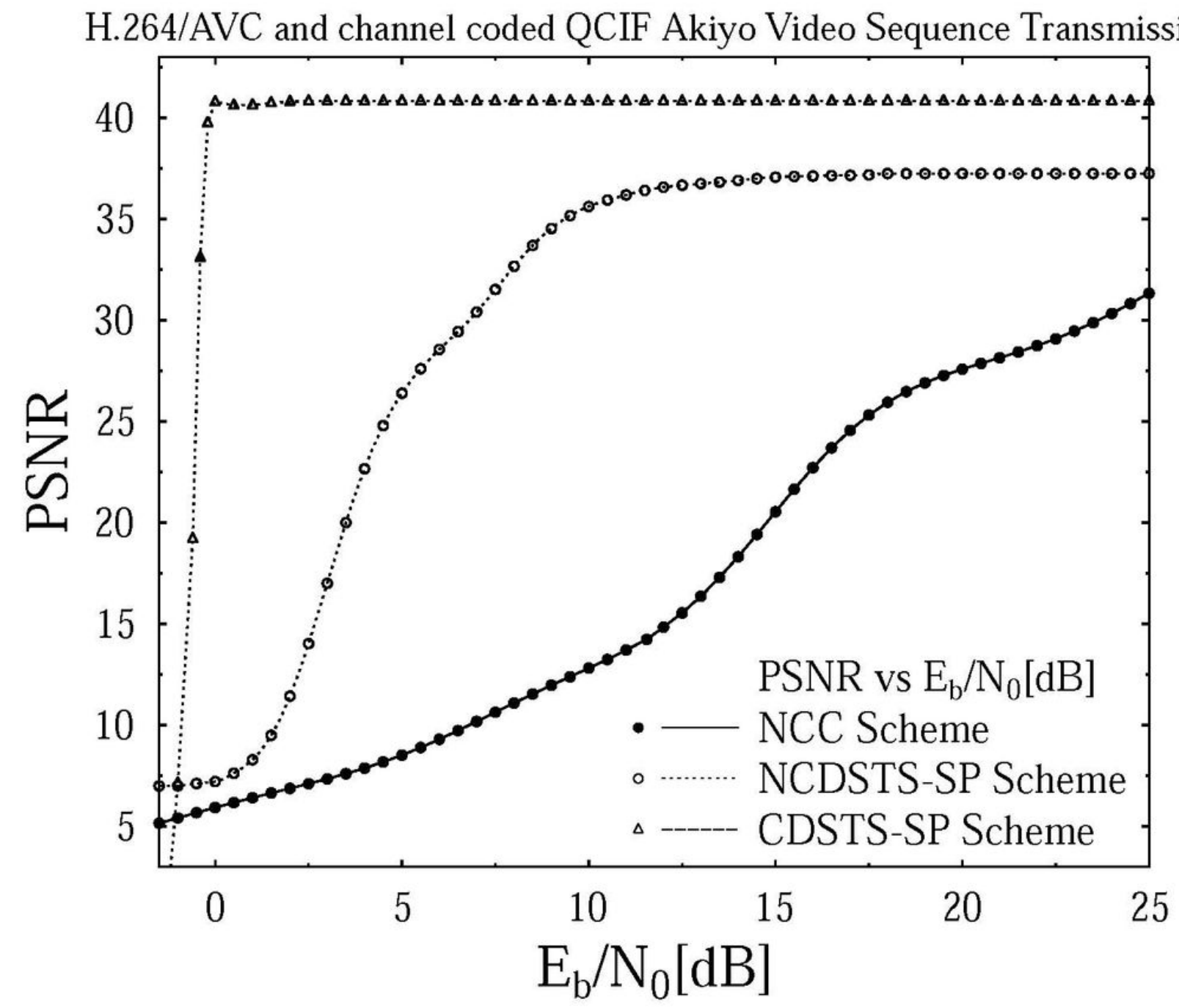

Figure 14

PSNR Vs Eb=NO for NCC, NCDSTS-SP and CDSTS-SP.

\section{Supplementary Files}

This is a list of supplementary files associated with this preprint. Click to download.

- bmcartbiblio.sty

- citesort.sty

- bmcarticle.bbl

- bmcarticle.bib

- bmcart.cls 
- bmcarticle.tex 